\title{
Roles of HIFs and VEGF in angiogenesis in the retina and brain
}

\author{
Amir Rattner, ${ }^{1}$ John Williams, ${ }^{1,2}$ and Jeremy Nathans ${ }^{1,2,3,4}$ \\ DDepartment of Molecular Biology and Genetics, ${ }^{2}$ Howard Hughes Medical Institute, ${ }^{3}$ Department of Neuroscience, and ${ }^{4}$ Wilmer Eye Institute, Johns Hopkins University School of Medicine, Baltimore, \\ Maryland, USA.
}

\begin{abstract}
Vascular development in the mammalian retina is a paradigm for CNS vascular development in general, and its study is revealing fundamental mechanisms that explain the efficacy of antiangiogenic therapies in retinal vascular disease. During development of the mammalian retina, hypoxic astrocytes are hypothesized to secrete VECF, which attracts growing endothelial cells as they migrate radially from the optic disc. However, published tests of this model using astrocyte-specific deletion of Vegf in the developing mouse retina appear to contradict this theory. Here, we report that selectively eliminating Vegf in neonatal retinal astrocytes with a Cfap-Cre line that recombines with approximately $100 \%$ efficiency had no effect on proliferation or radial migration of astrocytes, but completely blocked radial migration of endothelial cells, strongly supporting the hypoxic astrocyte model. Using additional Cre driver lines, we found evidence for essential and partially redundant actions of retina-derived (paracrine) and astrocyte-derived (autocrine) VEGF in controlling astrocyte proliferation and migration. We also extended previous studies by showing that HIF-1 $\alpha$ in retinal neurons and HIF-2 $\alpha$ in Müller glia play distinct roles in retinal vascular development and disease, adding to a growing body of data that point to the specialization of these 2 hypoxiasensing transcription factors.
\end{abstract}

\section{Introduction}

Vascular development is a tightly orchestrated process that generates artery, vein, and capillary patterns and densities that are precisely calibrated to meet local tissue requirements. The master regulator of vascular development and homeostasis is VEGF, a secreted polypeptide that is produced by target tissues in response to hypoxia. VEGF activates receptors on vascular endothelial cells (ECs) to promote EC survival, proliferation, permeability, and taxis toward the VEGF source, essential steps in the production and maintenance of blood vessels (1). Transcription of the Vegf gene is controlled by HIFs, $\alpha-\beta$ heterodimeric transcription factors that are stabilized by tissue hypoxia, thereby functioning as intracellular oxygen sensors (2). HIF control of VEGF production ensures that vessel growth occurs where and when it is needed. Depending on the level of activation of the HIF/VEGF system and the developmental context, the result can be vascular growth, stabilization, or regression.

Vascular development in the retina has long served as a model for vascular development in general and CNS vascular development in particular, both because defects in the retinal vasculature are of clinical interest and because the retina has an unusually accessible and stereotyped vascular anatomy $(3,4)$. In most mammalian retinas, including those of mice and humans, 3 layers of capillaries supply the metabolic needs of the inner retina. The arteries and veins that feed the intraretinal vessels enter the

Conflict of interest: JN is a paid consultant for Cerevance and Atengen. Copyright: @ 2019, American Society for Clinical Investigation.

Submitted: December 6, 2018; Accepted: June 13, 2019; Published: August 12, 2019.

Reference information: J Clin Invest. 2019;129(9):3807-3820.

https://doi.org/10.1172/JCl126655. globe at the optic nerve and then branch as they extend radially along the inner surface of the retina, adjacent to the vitreous (here after, referred to as the vitreal surface of the retina). Smaller vessels extend perpendicularly into the retina and connect the surface vascular plexus to 2 tiers of intraretinal capillaries. The retinal pigment epithelium and the rod and cone photoreceptors in the outer retina are supplied by the choroidal circulation. Because the retina is a planar structure less than $0.5 \mathrm{~mm}$ in thickness, it is possible to view an entire retina, including all of its vasculature, in a flat mount preparation.

In the mouse, the retinal vasculature develops during the first 2 weeks of postnatal life $(5,6)$. The initial phase involves proliferation and radial migration of astrocytes on the vitreal surface of the retina, followed by proliferation and radial migration of ECs along the astrocyte network. Both cell types originate from the optic disc. Astrocytes invade the retina prenatally, and ECs follow at postnatal day 1 (P1) (7). The 2 intraretinal capillary plexuses develop first in the central retina, starting at approximately $\mathrm{P} 7$, and then progress to the far periphery over the next several days. Development of the outermost capillary plexus occurs first, followed by the development of the intermediate capillary plexus.

The mechanisms that govern the radial growth of the retinal vasculature have been intensively studied. Early observations showed (a) high levels of Vegf mRNA in retinal astrocytes ahead of the radially expanding vascular front and (b) low levels of Vegf mRNA in the more centrally located retinal astrocytes that are in contact with the developing vasculature (8). These observations, together with demonstrations that exploratory ECs (tip cells) that populate the vascular front have numerous filopodia and that ECs in culture migrate toward a source of VEGF (9), served as the foundations of a model in which tip cells behave much like neuronal 
A
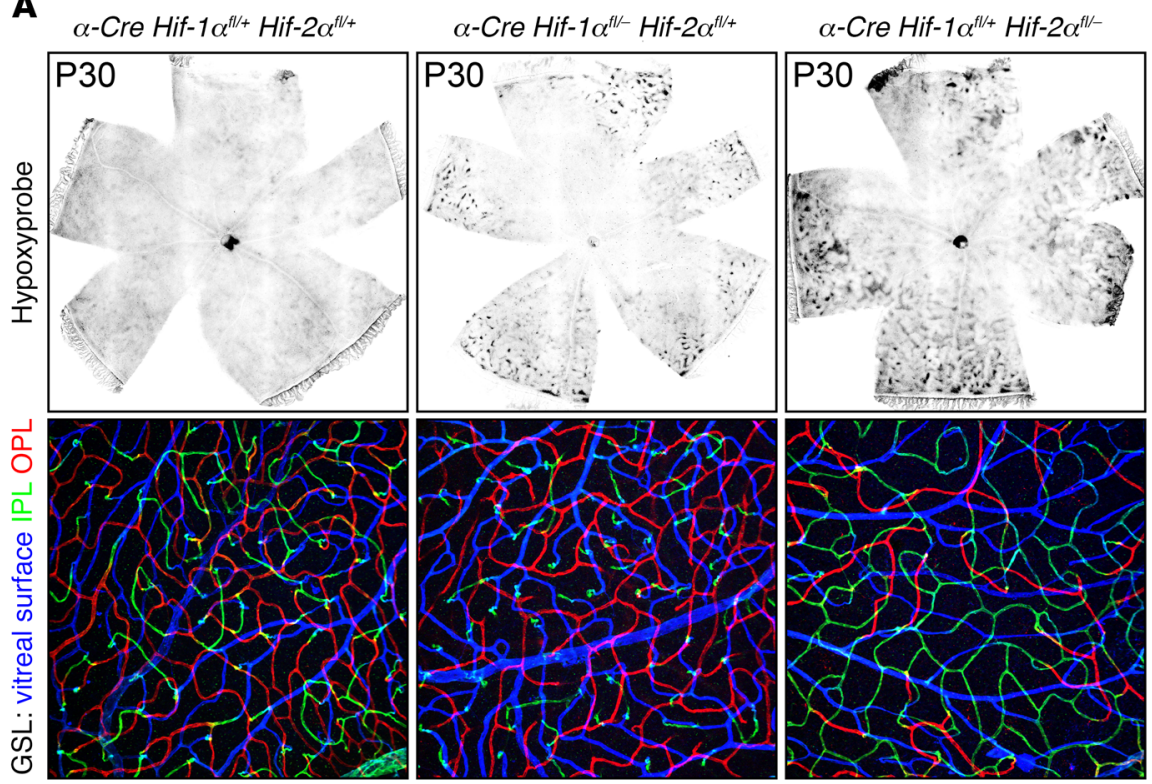

$\alpha$-Cre Hif- $1 \alpha^{t / /-}$ Hif- $2 \alpha^{t / /}$

B

- $\alpha$-Cre Hif- $1 \alpha^{f / /+}$ - $\alpha$-Cre Hif-2 $\alpha^{f l-}$
- $\alpha$-Cre Hif-1 $\alpha^{\text {t//- }}$

- $\alpha$-Cre Hif-2 $\alpha^{t /-}$

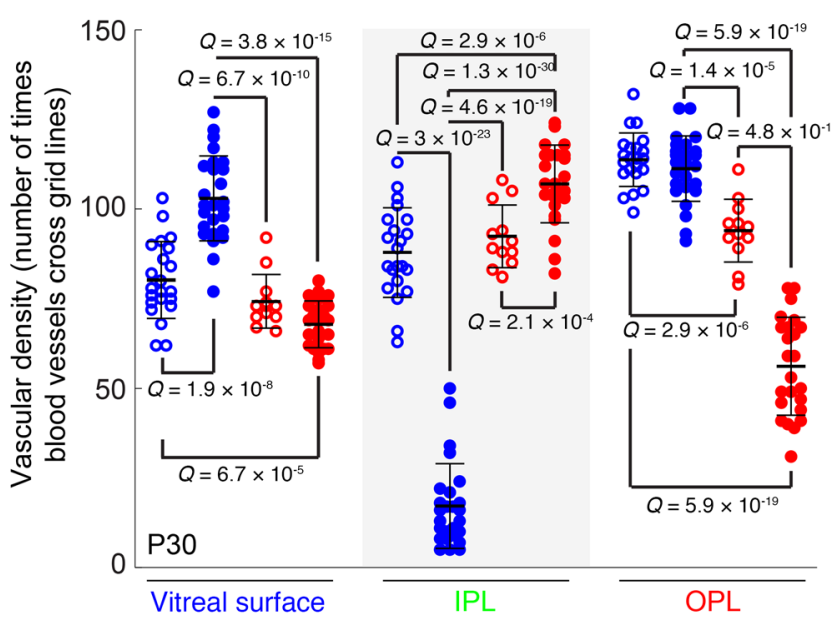

D

- Glast-Cre ${ }^{E R}$ Hif-1 $\alpha^{f / /+}$ Hif-2 $\alpha^{f /+} \circ$ o Glast-Cre ${ }^{E R}$ Hif-1 $\alpha^{f l /}$ Hif-2 $\alpha^{f / /+}$ - Glast-Cre ${ }^{E R}$ Hif-1 $\alpha^{f / /+}$ Hif-2 $\alpha^{f / /-}$ o Glast-Cre ${ }^{E R}$ Hif-1 $\alpha^{f / /}$ Hif-2 $\alpha^{\text {tl/- }}$
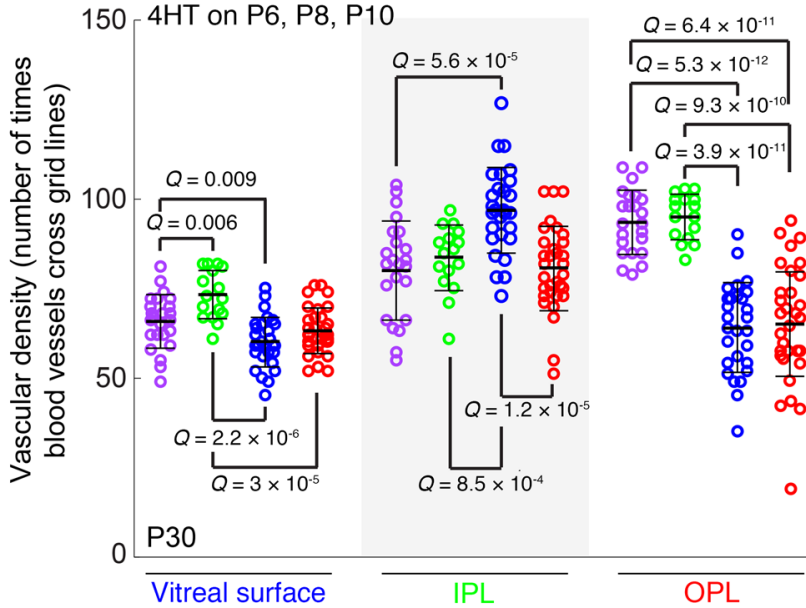

$00=3.9 \times 10^{-11}$
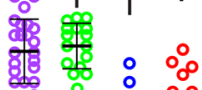
$\circ \frac{0}{80}$

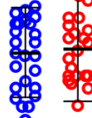

。
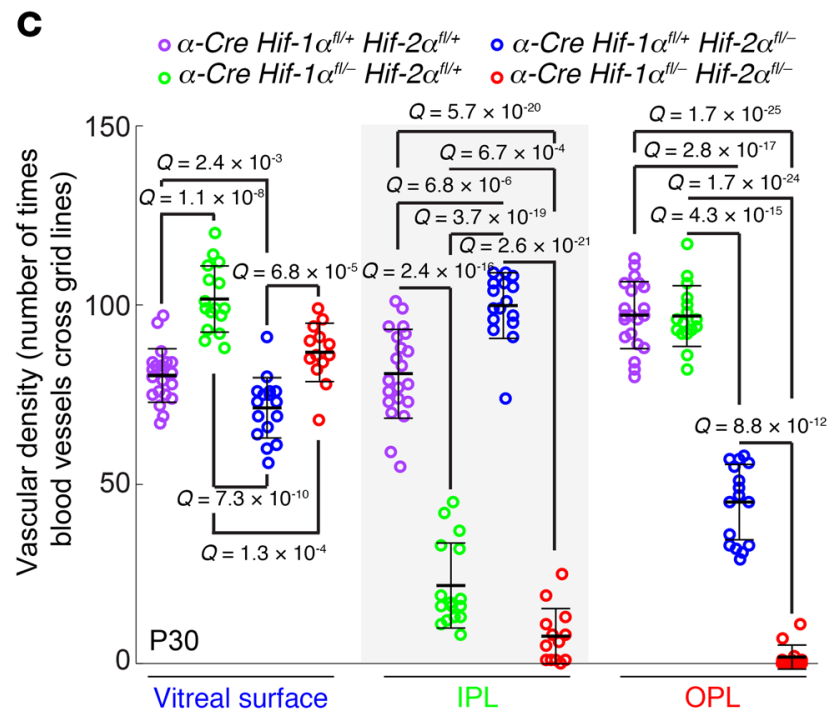

E

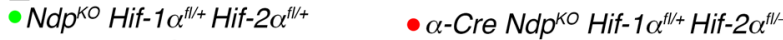

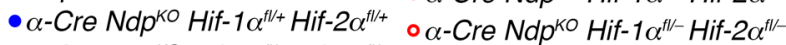
- $\alpha$-Cre Ndp ${ }^{K O}$ Hif-1 $\alpha^{\text {Il/ }}$ Hif-2 $\alpha^{i / /+}$

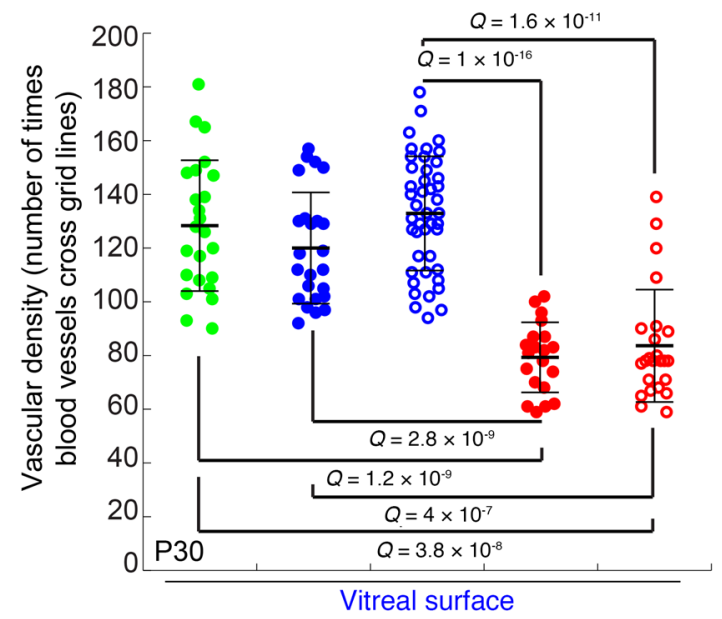


Figure 1. Distinctive vascular phenotypes result from eliminating Hif$1 \alpha$, Hif-2 $\alpha$, or both Hif-1 $\alpha$ and Hif-2 $\alpha$ in developing retinal progenitors or Müeller glia. (A) Top panels, P30 whole retina flat mounts from the indicated genotypes stained for Hypoxyprobe (black), which accumulates in hypoxic regions of retina. The $\alpha$-Cre Hif- $1 \alpha^{f l /+} H i f-2 \alpha^{f / /+}$ retina (left panel) is a phenotypically normal control. Scale bar, $1 \mathrm{~mm}$. Bottom panels, highmagnification images from the peripheral retina, where the $\alpha$-Cre transgene is expressed, showing GSL-stained vasculature. The GSL signal is color coded by depth with the vitreal surface in blue, the IPL in green, and the OPL in red. Scale bar: $200 \mu \mathrm{m}$. (B-D) Quantification of the vascular density in the peripheral retina for each of the 3 vascular layers (vitreal surface, IPL, and $\mathrm{OPL}$ ) following $\alpha$-Cre (B and C) or Glast-CreER (D) mediated deletion of the indicated Hif- $1 \alpha$ and/or Hif-2 $\alpha$ alleles. Each symbol represents the quantification from one $500-\mu \mathrm{m} \times 500-\mu \mathrm{m}$ square area. For activation of CreER in the Glast-CreER mice, each mouse received 3 doses of $200-\mu \mathrm{g} 4 \mathrm{HT}$, delivered at P6, P8, and P10. In this and all other figures; data show the mean $\pm S D$, and $Q$ values were calculated with a 2 -tailed unequal variance $t$ test adjusted for multiple comparisons using the method of Benjamini and Hochberg (52). (E) Quantification of the surface vascular density in the peripheral retina following $\alpha$-Cre-mediated recombination of the indicated Hif- $1 \alpha$ and/or Hif- $2 \alpha$ alleles in an Ndp ${ }^{K O}$ background. Ndp is X-linked and the $N d p^{K 0}$ retinas studied here were hemizygous for the KO allele. Each symbol represents the quantification from one $500-\mu \mathrm{m} \times 500-\mu \mathrm{m}$ square area.

growth cones, attracted by the VEGF that is secreted from hypoxic astrocytes. In this model, vascular growth is guided by physiologic hypoxia, first in astrocytes ahead of the growing vascular plexus, and then, later in development, in other cell types within the retina to guide development of the intraretinal capillaries $(8,10)$. In support of the intraretinal phase of this model, recent experiments have shown that activating or suppressing the HIF/VEGF system in horizontal or amacrine cells - interneurons that are immediately adjacent to the 2 tiers of intraretinal capillaries - leads to a corresponding enhancement or reduction, respectively, in the density of the adjacent capillary plexus (11).

An incisive test of the hypoxic astrocyte model of radial EC migration would be to eliminate the HIF/VEGF system selectively in astrocytes and determine whether this also eliminates EC migration. Such a test has been reported by 3 groups. Two groups report little or no effect on EC migration following Cre-mediated disruption of the Vegf gene in astrocytes $(12,13)$. The third group reports that Cre-mediated disruption of the Hif-2a gene affects both astrocyte and EC migration, leaving open the question of whether the EC migration defect is referable to a decrement in astrocyte-derived VEGF (14). As a result of these experiments, the current consensus in the field is that astrocyte-derived VEGF plays little or no role in guiding radial migration of ECs during normal retinal development $(3,15)$.

In the present study, we have explored the HIF/VEGF model of retinal vascular development in the mouse using conditional loss-of-function analyses of Hif- $1 \alpha$ and Hif- $2 \alpha$, both separately and in combination, and of Vegf, with the goal of defining the roles of individual cell types and of the 2 HIF- $\alpha$ subunits. Importantly, we have used Cre drivers that are well defined with respect to cell type specificity and recombination efficiency. The results provide clear evidence in favor of the original HIF/VEGF model, define distinctive divisions of labor among retinal cells and HIF- $\alpha$ proteins, and suggest a similar paradigm in the context of cerebral cortical vascular development.

\section{Results}

Distinct roles for HIF-1 $\alpha$ and HIF-2 $\alpha$ in the development of intraretinal capillaries. HIF-1 $\alpha$ and HIF-2 $\alpha$ play essential roles in the development of inner plexiform layer (IPL) and outer plexiform layer (OPL) capillaries, respectively $(11,16,17)$. To confirm and extend these observations, we began by quantitatively assessing

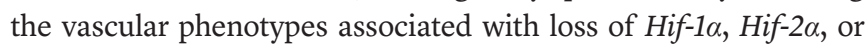
both Hif-1 $\alpha$ and Hif-2 $\alpha$ in retinal neurons and Müller glia (Figure $1, \mathrm{~A}-\mathrm{C})$. In these experiments, conditional alleles of Hif-1 $\alpha$ and Hif-2 $\alpha$ were inactivated by $\mathrm{Cre}$-mediated recombination in retinal progenitor cells at approximately embryonic day 9 (E9) using the Pax6 $\alpha$-Cre transgene (hereafter referred to as $\alpha$-Cre; ref. 18). The expression of this transgene is confined to the nasal and temporal sides of the peripheral retina, leaving an unrecombined dorsoventral stripe in the center of the retina as an internal control. $\alpha$-Cre does not recombine in surface astrocytes or ECs (19). To maximize the efficiency with which Cre-mediated recombination produces complete loss of gene function, $\mathrm{fl} /$-rather than $\mathrm{fl} / \mathrm{fl}$ genotypes were studied, so that only a single recombination event was required to convert a heterozygous cell to a homozygous null cell. In these experiments, we assessed the phenotypes associated with heterozygous versus homozygous loss of a single Hif- $\alpha$ gene ( $\alpha$-Cre Hif-1 $\alpha^{f / /+}$ vs. $\alpha$-Cre Hif- $1 \alpha^{f / /-}$ and $\alpha$-Cre Hif-2 $2 \alpha^{f /+}$ vs. $\alpha$-Cre Hif-2 $\alpha^{f / /}$; Figure 1B), as well as all combinations of heterozygous and homozygous loss of Hif-1 $\alpha$ and Hif-2 $\alpha$. The latter genotypes were generated in a cross between $\alpha$-Cre Hif- $1 \alpha^{+/-}$Hif-2 $\alpha^{+/-}$and Hif-1 $1 \alpha^{A / f l}$ Hif-2 $2 a^{A / f l}$ mice.

In flat-mount preparations of retina, the extent and location of hypoxia was visualized by in vivo labeling with Hypoxyprobe (pimonidazole), a small molecule that is administered by i.p. injection and accumulates in hypoxic cells, where it forms covalent adducts (Figure 1A and ref. 20). In the same flat mount preparations, vascular structure was visualized by GS-lectin staining, and vascular density was quantified for each of the 3 tiers of vessels -1 on the vitreal surface and a pair that flank the inner nuclear layer (INL), the IPL, and OPL capillary beds-which are color coded in blue, green, and red, respectively, in Figure 1A.

In assessing statistical significance in Figure 1 and in subsequent figures, each data point in the scatter plot represents an individual image obtained from a separate quadrant of the retina, and the $Q$ values ( $P$ values adjusted for multiple tests) shown reflect those data points. For every scatter plot, we have also calculated $Q$ values for data points aggregated on a perretina basis and on a per-mouse basis. These 2 sets of $Q$ values, together with the $Q$ values calculated for the individual images, are listed in Supplemental Tables 1-9 (supplemental material available online with this article; https://doi.org/10.1172/ JCI126655DS1.), which also lists the sample sizes for each experiment and for each grouping of data points. See the Methods section for further details.

In comparing WT retinas to retinas with heterozygous loss of either or both Hif- $\alpha$ genes, no statistically significant differences $(\mathrm{Q}<0.05)$ were seen in the density of vessels on the vitreous face, and no statistically significant differences in intraretinal vascular density were seen in comparisons of WT versus the 2 single-gene heterozygotes. However, for the WT versus double heterozygote comparison, the IPL density is lower in the double heterozygote, 
A

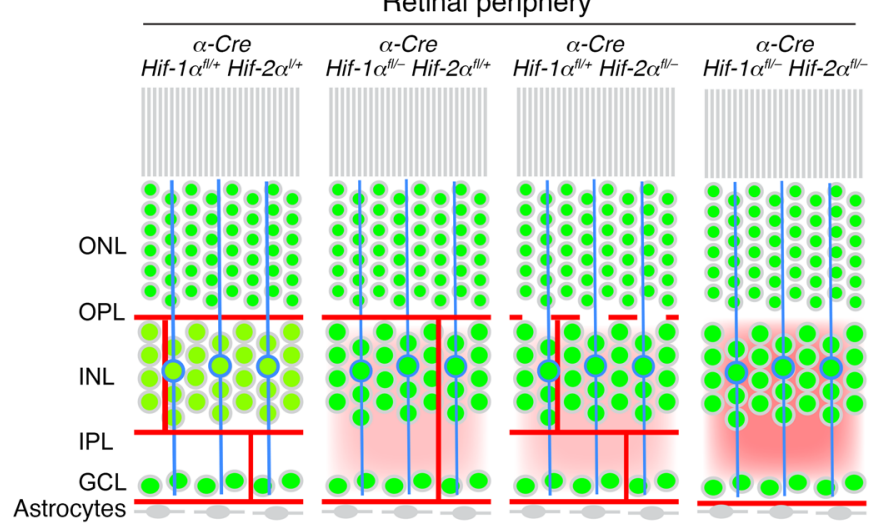

B

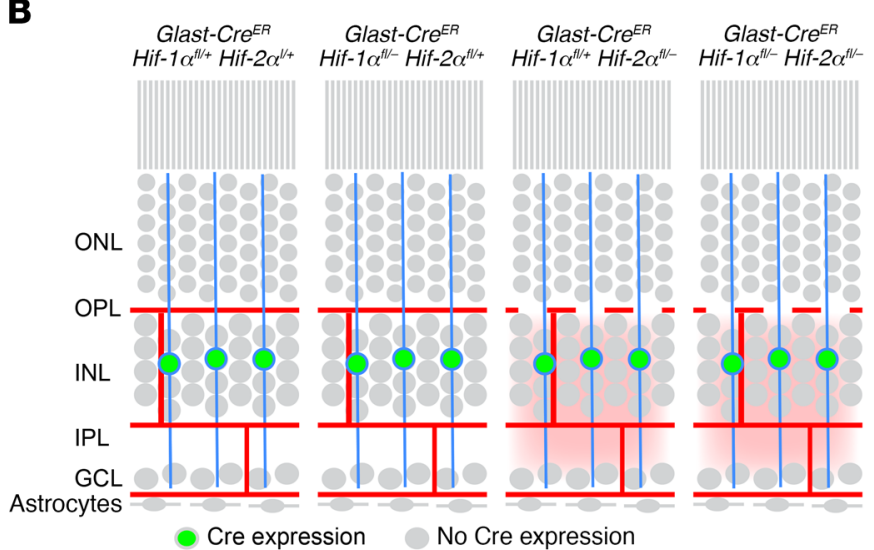

and this difference is statistically significant if one calculates $Q$ values based on individual images, although this trend fails to reach statistical significance if the calculation is performed with individual retinas or individual mice (Figure 1, A and C; Supplemental Figure 1; and Supplemental Table 9). These data imply a subtle dose dependence of Hif- $\alpha$ gene function, as revealed by the double heterozygous phenotype.

Homozygous loss of Hif-1 $\alpha$ produced an approximately 4 -fold reduction in the density of IPL capillaries, and homozygous loss of Hif-2 $\alpha$ produced an approximately 2 -fold reduction in the density of OPL capillaries (Figure 1, A-C). For each genotype, the remaining vascular layers were minimally or not at all affected. Loss of IPL capillaries in the homozygous Hif-1a KO was accompanied by a modest hypertrophy of vessels on the vitreal surface of the retina, presumably as a response to hypoxia in the adjacent inner retinal cells.

Strikingly, homozygous loss of both Hif- $1 \alpha$ and Hif- $2 \alpha$ led to a nearly complete absence of both OPL and IPL vasculature. The severity of the double mutant phenotype implies that the proangiogenic signals controlled by these 2 factors are partially redundant and absolutely required for intraretinal vascularization. The extent and location of tissue hypoxia as seen by Hypoxyprobe labeling correlated closely with the anatomic patterns of vascular deficiency, with reductions in the individual OPL and IPL capillary beds producing modest hypoxia and the complete loss of both OPL and IPL capillary beds producing severe hypoxia (Figure 1A).
C
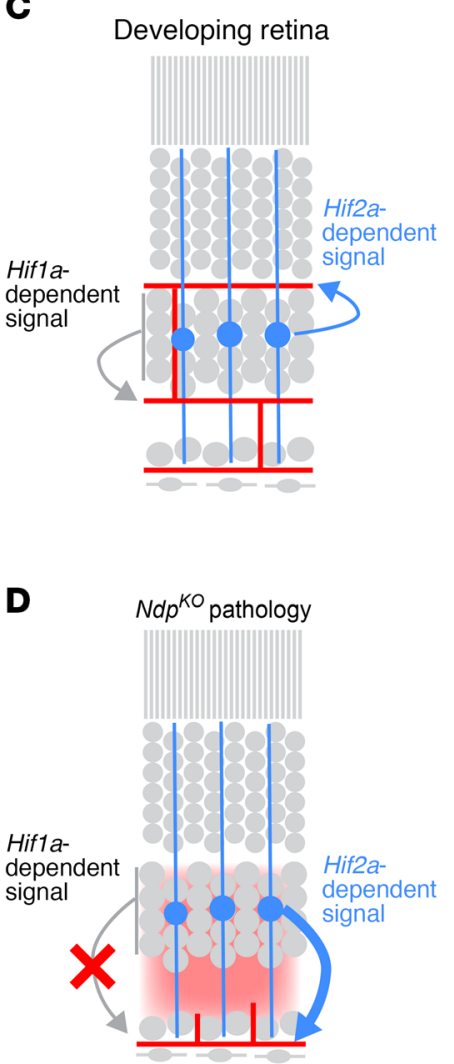

Figure 2. Complementary roles for Hif- $1 \alpha$ and Hif-2 $\alpha$ in the development of intraretinal capillaries. (A and $\mathbf{B})$ The principal cell layers of the adult retina, with neuronal and astrocyte cell bodies (grey), Müller glia cell bodies and processes (blue circles and vertical lines), retinal vasculature (red lines), extent of hypoxia (red background), and presence of Cre-mediated recombination (green circles; representing Cre activation of a nuclear localized GFP reporter). Summary phenotypes are shown for $\alpha$-Cre (A) or Clast-CreER (B) mediated deletion of the indicated Hif- $1 \alpha$ and/or Hif-2 $\alpha$ alleles. (C and D) Retinal schematics as in $\mathbf{A}$ and $\mathbf{B}$ showing the cellular sources, vascular targets, and relative strengths of Hif-1 $\alpha$-dependent and Hif-2 $\alpha$ dependent proangiogenic signals during the final phase of retinal development (C) and in response to hypoxia in the mature $\mathrm{Ndp} \mathrm{K}^{\mathrm{O}}$ retina (D).
As an initial step in defining the cell type(s) in which Hif-1a and Hif-2 $\alpha$ act, we used a Glast-CreER transgene and a regimen of i.p. 4-hydroxytamoxifen (4HT) injections at P6, P8, and P10 to catalyze gene deletion specifically in Müller glia just prior to and partially overlapping with the time during which IPL and OPL capillaries are developing (P8-P15; refs. 21 and 22). These genotypes were generated in a cross between Glast-CreER Hif-1 $\alpha^{+-}$ Hif-2 $\alpha^{+/}$, and Hif-1 $\alpha^{f / / f l} H i f-2 \alpha^{f / f l}$ mice. Figure 1D shows that Müller glia-specific deletion of Hif-1 $\alpha$ in combination with heterozygous loss of Hif-2 $\alpha$ had no effect on the development of the IPL or any other part of the retinal vasculature. By contrast, Müller gliaspecific deletion of $H i f-2 \alpha$ in combination with heterozygous loss of Hif-1 $\alpha$ or combined deletion of Hif-1 $\alpha$ and Hif-2 $\alpha$ led to an approximately $40 \%$ reduction in OPL vascular density with no effect on IPL vascular density. The more modest reduction in capillary density observed with Glast-CreER-mediated compared with $\alpha$-Cremediated gene deletion (compare Figure 1, C and D), likely arises from the partial overlap in time between $4 \mathrm{HT}$ administration and OPL capillary development ( P8-P12) and/or the less-thancomplete recombination typically produced by $4 \mathrm{HT}$ and CreER. By contrast, the failure to observe an IPL defect in mice lacking Hif-1 $\alpha$ in Müller glia is probably not due to any temporal overlap between Cre-mediated recombination and capillary development, as the IPL capillary plexus develops 2-3 days later than the OPL plexus. The simplest explanation for the data in Figure 1, A-D is that HIF-1 $\alpha$ functions in retinal neurons but not in Müller glia and 
A

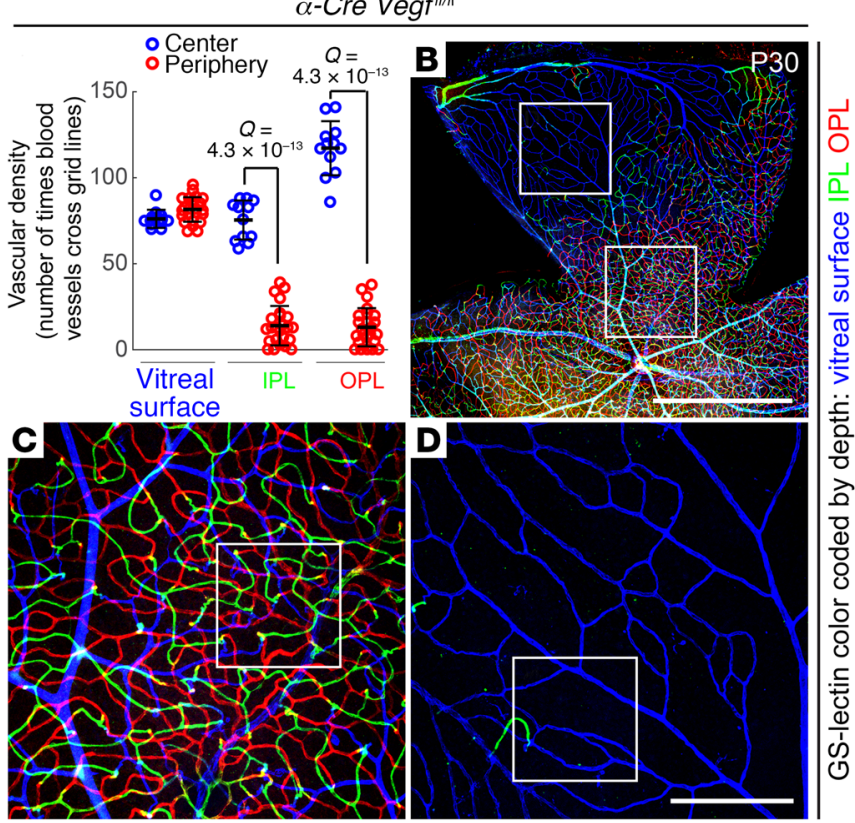

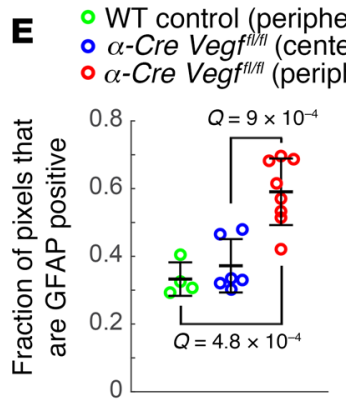

$\alpha$-Cre Vegffl/fl (center)

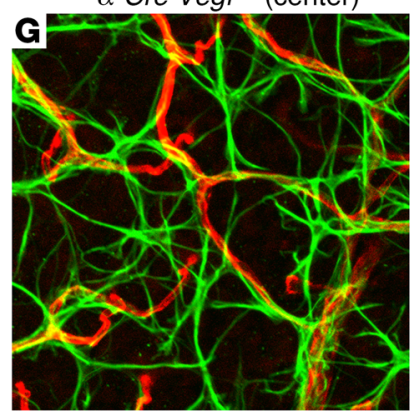

WT control (periphery)

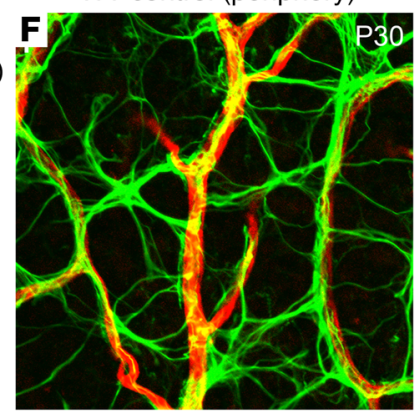

$\alpha$-Cre Vegft/th (periphery)

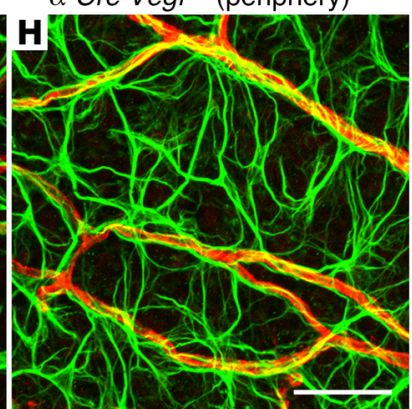

that HIF-2 $\alpha$ functions in Müller glia but not in neurons, consistent with the immunohistochemical analysis of Mowat et al. (23).

In an earlier study of retinas that lack Norrin/Frizzled 4 signaling and fail to develop IPL and OPL capillary beds ( $N d p^{K O}$ retinas; $N d p$ is the gene coding for Norrin), we showed that (a) deleting Hif-2 $\alpha$ with $\alpha$-Cre substantially reduced the compensatory hyperproliferative response of the vasculature on the vitreal surface of the retina, (b) this response was similarly reduced following deletion of Hif-2 $\alpha$ selectively in Müller glia (with Glast-CreER), and (c) the hyperproliferative response was likely mediated by HIF-2 $\alpha$ induction of VEGF in Müller glia (22). Figure 1E shows that deleting Hif-1 $\alpha$ with $\alpha$-Cre has no detectable effect on proliferation of the surface vasculature in $N d p^{K O}$ retinas.
Figure 3. Loss of intraretinal but not surface vasculature with loss of intraretinal VEGF. (A-D) Vascular density in $\alpha$-Cre Vegf $f^{f / f l}$ retinas at P30. (A) Quantification of vascular density in the center (blue; little or no Cre-mediated recombination) and periphery (red; intraretinal Cre-mediated recombination) for each of the 3 vascular layers (vitreal surface, IPL, and $\mathrm{OPL})$ in $\alpha$-Cre Vegfflfl retinas. In A, each symbol is the quantification from one $500-\mu \mathrm{m} \times 500-\mu \mathrm{m}$ area; bars represent the mean \pm SD and $\underline{Q}$ values were calculated with a 2 -tailed unequal variance $t$ test adjusted for multiple comparisons using the method of Benjamini and Hochberg (52). (B-D) GSL-stained vasculature at low magnification (B) and at intermediate magnification (C) and (D), corresponding, respectively, to the lower and upper labeled squares in $\mathbf{B}$. The CSL signal is color coded by depth with the vitreal surface in blue, the IPL in green, and the OPL in red. Scale bars: $1 \mathrm{~mm}$ (B) and $200 \mu \mathrm{m}$ (D). (E-H) Surface astrocyte density in $\alpha$-Cre Vegf fl/fl retinas at P30. E shows the quantification of the density of astrocyte processes (GFAP immunostaining) in the periphery of a WT retina (green) and in the center (blue) and periphery (red) of $\alpha$-Cre Vegf fl/fl retinas. Each symbol is the quantification from one $200-\mu \mathrm{m} \times 200-\mu \mathrm{m}$ area; bars represent the mean \pm SD, and $Q$ values were calculated with a 2 -tailed unequal variance $t$ test adjusted for multiple comparisons using the method of Benjamini and Hochberg (52). Examples of the vitreal surface of WT peripheral retina and $\alpha$-Cre Vegf $f^{\text {I/fl }}$ central and peripheral retina following GFAP and GSL staining are shown at high magnification in $\mathbf{F}-\mathbf{H}$ (scale bar: $50 \mu \mathrm{m}$ ). Images in $\mathbf{G}$ and $\mathbf{H}$ correspond to the labeled squares in $\mathbf{C}$ and $\mathbf{D}$, respectively.

Moreover, deletion of both Hif- $1 \alpha$ and Hif-2 $\alpha$ leads to the same reduction in the proliferation of surface vasculature as deletion of 1 allele of Hif- $1 \alpha$ and both alleles of Hif- $2 \alpha$. These data support a model in which HIF-1 $\alpha$ makes little or no contribution to the proangiogenic response in $N d p^{K O}$ retinas, despite the essential role of HIF-1 $\alpha$ in the development of IPL capillaries.

Figure 2 summarizes the experiments shown in Figure 1. The severity and location of tissue hypoxia (in red) is inferred from Hypoxyprobe staining and from the vascular anatomy associated with each genotype. Müller glia are represented by the 3 blue cell bodies in the INL with vertical cytoplasmic extensions. Green nuclei represent cells in which $C r e$-mediated recombination has occurred. Panels A and B summarize the effects of various gene deletions with $\alpha$-Cre or GLAST-CreER, respectively. Panels C and $\mathrm{D}$ summarize the sources, vascular targets, and magnitudes of HIF- $1 \alpha$ - and HIF- $2 \alpha$-dependent proangiogenic signals in WT and $N d p^{K O}$ retinas. The data presented here extend previous studies $(16,23,24)$ and show that HIF- $1 \alpha-$ and HIF- $2 \alpha$-dependent signals originate in different retinal cells, target different vascular beds, and respond differently to pathologic hypoxia.

Retina-derived VEGF: essential role in the growth of intraretinal capillaries but not surface vessels. As noted in the Introduction, a large body of evidence has shown that, in the retina, VEGF is the dominant mediator of the hypoxia-driven proangiogenic response both in normal development and in the context of pathologic hypoxia. However, the functions associated with different retinal sources of VEGF remain controversial. As a first step in addressing this finding, we studied the phenotype associated with eliminating retinal VEGF with $\alpha$-Cre, which, as noted previously, recombines target genes in retinal neurons and Müller glia but not in astrocytes or ECs (19).

In retinas from $\alpha$-Cre Vegfl/fl mice, the vasculature in the central retina is unaffected (Figure 3, A-C), but in the zones of $\alpha$-Cre expression in the peripheral retina, the only vasculature present is on the vitreal surface (Figure 3, A, B, and D). These data imply that 
Gfap-Cre R26-LSL-mtdT-2A-H2B-GFP

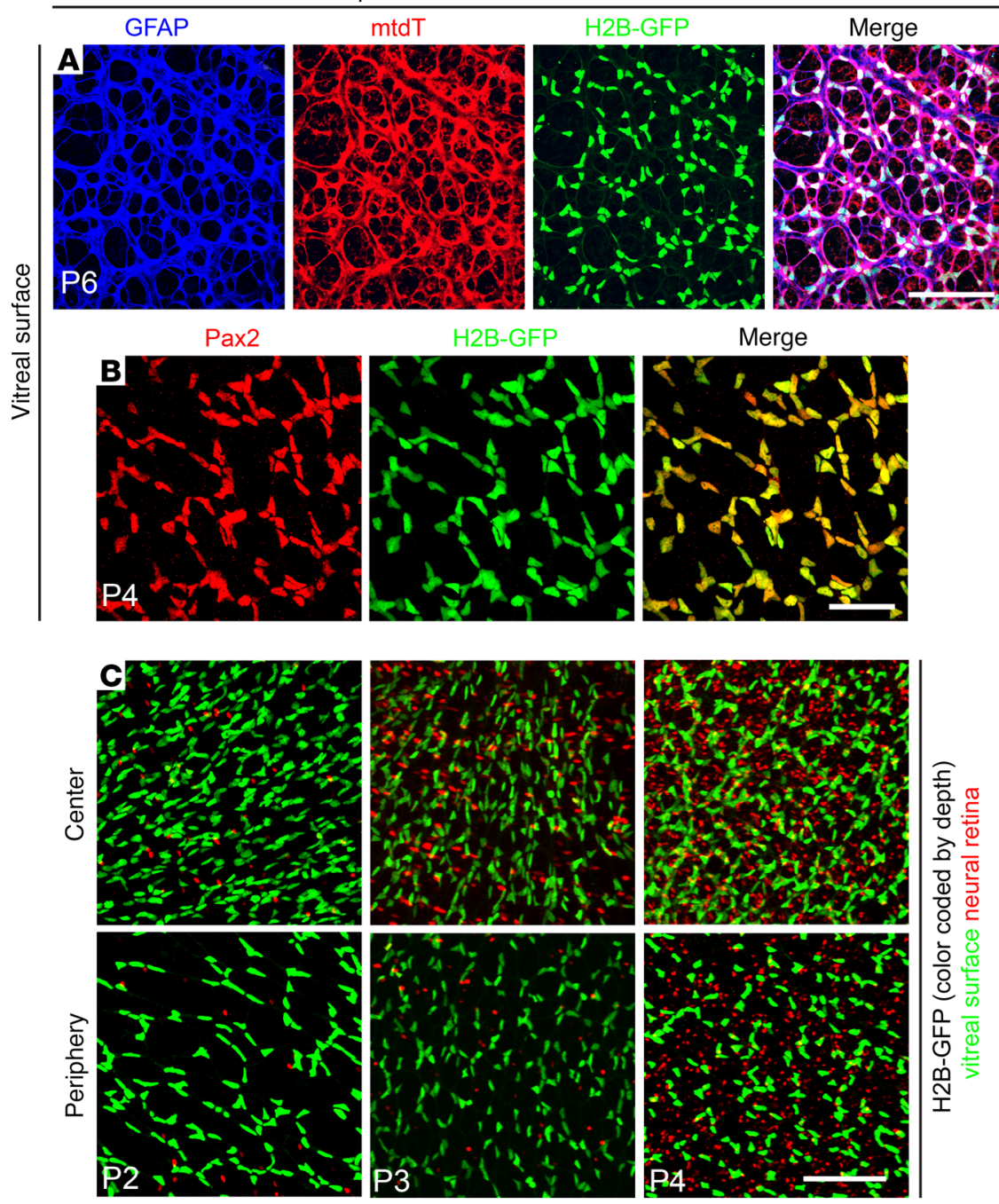

Figure 4. Highly efficient Cre-mediated recombination in early postnatal retinal astrocytes by the Messing Cfap-Cre line, as seen in retina flat mounts from Gfap-Cre R26-LSL-mtdT-2A-H2B-CFP retinas between $\mathbf{P 2}$ and P6. (A and $\mathbf{B})$ Retinas imaged the vitreal surface and showing GFAP, mtdT, and H2B-GFP at P6 (A) and Pax2 and H2B-GFP at P4 (B). (C) H2B-GFP at the vitreal surface (green) and in the neural retina (red) at P2, P3, and P4. Highly efficient recombination in surface astroctyes is followed by scattered recombination in cells that are deeper within the neural retina. See Figure 7A for a cross-sectional view. Scale bars: $100 \mu \mathrm{m}$ (A), $50 \mu \mathrm{m}$ (B), and $100 \mu \mathrm{m}$ (C).

Visualizing ECs and surface astrocytes in the periphery of WT and $\alpha$-Cre Vegfl/fl retinas and in the phenotypically WT center of $\alpha$-Cre $V e g f^{f / f l}$ retinas shows the normal association of ECs and astrocytes and also reveals an approximately 2-fold increase in the density of astrocyte processes in the affected regions of $\alpha$-Cre Vegfl/fl retinas (Figure 3, E-H). This density increase presumably represents a homeostatic response to local hypoxia that occurs in the absence of locally synthesized retinaderived VEGF.

Astrocyte-derived VEGF: essential role in radial migration of surface ECs. The $\alpha$-Cre $V e g f^{l / f l}$ experiments described in the preceding paragraphs argue against a trophic role for retinal neuron- and/or Müller glia-derived VEGF during radial growth of the surface vasculature. Instead, they are consistent with the classic studies of Stone, Keshet, and Gerhardt, along with other studies that implicated hypoxic astrocytes ahead of the growing vascular front as the source of a trophic VEGF gradient that attracts ECs. As described in the Introduction, this model has fallen out of favor because 2 independent groups reported that knocking out Vegf in retinal astrocytes using Gfap-Cre Vegfl/fl mice had little or no effect on radial migration of developing ECs $(12,13)$.

One potential caveat in the published Gfap-Cre Vegfl/fl experiments is the possibility that Cre-mediated recombination in astrocytes may have been incomplete. Multiple Gfap-Cre lines exist (see http://www.informatics.jax. org/recombinase/summary?driver=GFAP), and these lines exhibit a range of expression patterns and recombination efficiencies. Both Scott et al. (12) and Weidemann et al. (13) used the Gfap-Cre line generated by the Gutmann lab, Tg (GFAP-Cre) 8Gtm (25). In contrast, Duan et al. (2014) used the Gfap-Cre line generated by the Messing lab, Tg (Gfap-Cre) 25Mes/J

the radial growth and subsequent maintenance of the surface vasculature does not require local retina-derived VEGF. We note that this experiment does not exclude the possibility that VEGF derived from the central retina acts at a distance to promote growth and maintenance of the surface vasculature in the retinal periphery.

Despite the absence of intraretinal capillaries in the affected regions of $\alpha$-Cre $\mathrm{Veg} f^{\mathrm{fl} / \mathrm{fl}}$ retinas, there is no compensatory hypertrophy of the surface vasculature. This finding is in contrast to the hypertrophy of the surface vasculature observed in $N d p^{K O}$ retinas, which similarly lack intraretinal capillaries. The experiments in Figures 1 and 3, together with early experiments showing strong VEGF induction specifically in Müller glia in $N d p^{K O}$ retinas (22), support a model in which Müller glia-derived and HIF-regulated VEGF are the principal mediators of the proangiogenic response in the context of reduced intraretinal capillary density.
(26) to delete Hif- $2 \alpha$ in retinal astrocytes and observed a severe defect in radial migration of ECs. Duan et al. (14) interpreted this phenotype in the context of the published Gfap-Cre Vegfl/fl results and suggested that the EC migration defect might arise from an undetermined defect in astrocyte-EC interactions. However, an alternative explanation is that the Messing Gfap-Cre line catalyzes more efficient Cre-mediated recombination in astrocytes than the Gutmann Gfap-Cre line that was used in the 2 published Gfap-Cre $V e g f^{f / f l}$ studies, thereby producing a complete or nearly complete loss of astrocyte Hif- $2 \alpha$ and secondarily an absence of hypoxiainduced Vegftranscription. To test this alternative explanation, we compared the timing and efficiency of Cre-mediated recombination in the $\mathrm{Tg}$ (GFAP-Cre) 8Gtm and $\mathrm{Tg}$ (Gfap-Cre) 25Mes/J lines.

A loxP-stop-loxP (LSL) reporter knockin at the Rosa26 locus was used to assess the efficiency and cell type specificity of 
A
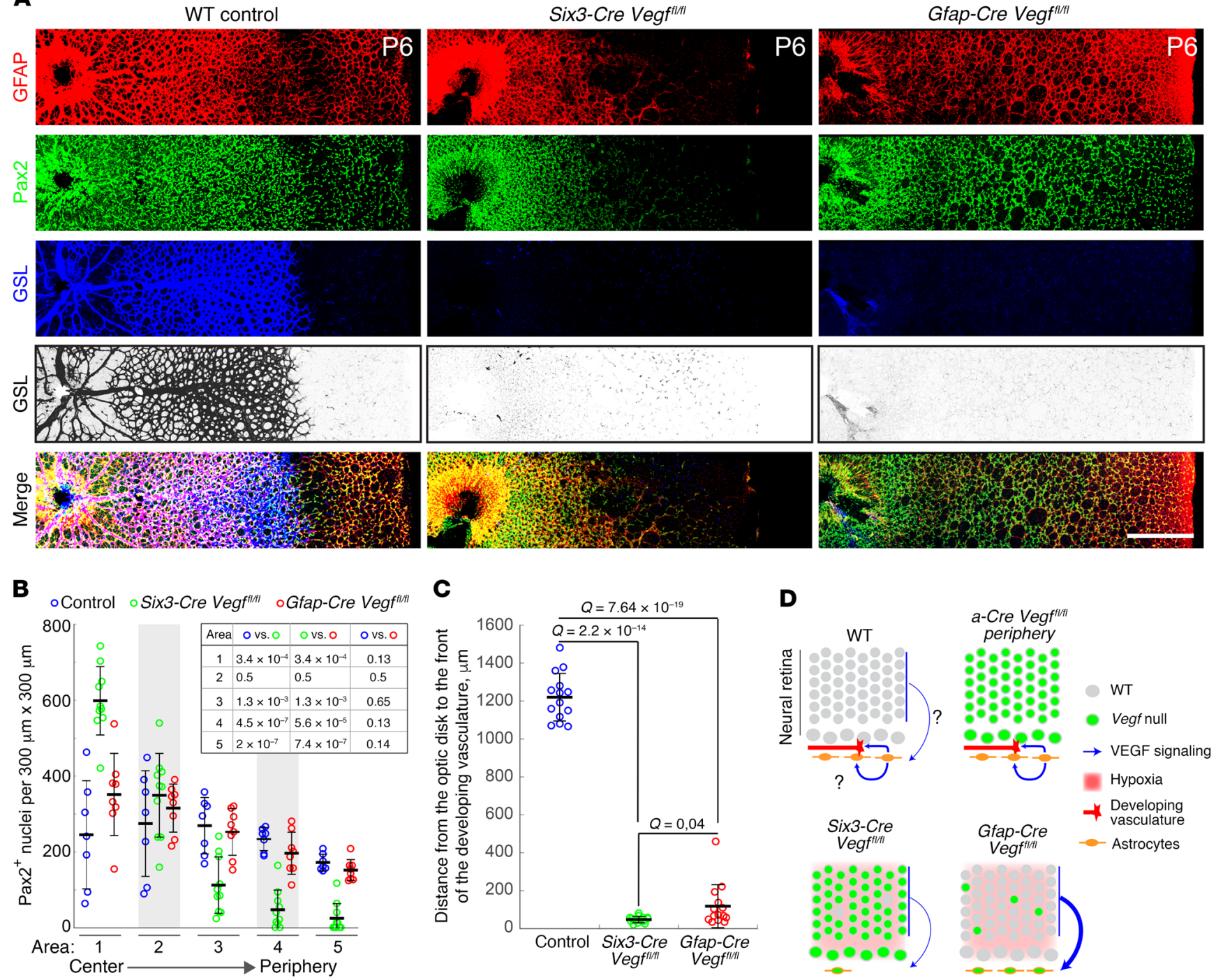

Figure 5. Loss of astrocyte-derived VEGF eliminates radial EC migration during retinal development. (A) Flat mounts of WT, Six3-Cre Vegf $f^{f / / f l}$, and Gfap-Cre Vegf $f^{f / f l}$ retinas at P6, immunostained for astrocytes (GFAP and Pax2) and ECs (GSL). The latter is shown both in color and, for further clarity, in black and white with intensities inverted. The optic disc is at the left of each image, and the peripheral edge of the retina is at the far right. Scale bar: $400 \mu \mathrm{m}$. (B) Quantification of astrocyte radial migration at P6. The number of Pax2 $2^{+}$nuclei was counted in radial strips divided into five contiguous $300-\mu \mathrm{m} \times 300-\mu \mathrm{m}$ squares, from the retinal center to the periphery. Each symbol shows the Pax2 $2^{+}$nuclear counts from 1 square; data show the mean \pm SD. Seven to 10 radial strips were quantified per genotype. $Q$ values for pairwise comparisons, calculated using 2-tailed unequal variance $t$ test adjusted for multiple comparisons using the method of Benjamini and Hochberg (52), are presented in the inset table. (C) Quantification of EC radial migration at P6. The radial distance from the center of the retina to the growing vascular front (based on CSL staining) was measured on 4 to 8 retinas per genotype, for a total of 14 measurements per genotype. Data show the mean $\pm \mathrm{SD}$, and $Q$ values were calculated with a 2-tailed unequal variance $t$ test adjusted for multiple comparisons using the method of Benjamini and Hochberg (52). (D) Schematics of early postnatal retinas showing the cellular sources, vascular targets, and relative strengths of VEGF signaling inferred from the phenotypes of retinas with the indicated genotypes. Symbols are defined to the right of the 4 schematics. A question mark indicates uncertainty regarding the existence or strength of a VEGF signal. The neural retina is shown as it appears at approximately $\mathrm{P} 3$, with a ganglion cell layer that is distinct from an outer layer encompassing neuroblasts and future INL/ONL cells. Representative images are shown from experiments with 4 or more eyes per genotype.

Cre-mediated recombination. The reporter, R26-LSL-mtdT-2A$H 2 B-G F P$, expresses a membrane-associated tdTomato (tdT) and a nuclear-localized H2B-GFP following Cre-mediated recombination (27). Flat mounts of P4 to P6 retinas show a nearly perfect correlation between cells at the vitreal surface of the retina expressing (a) tdT and the astrocyte cytoplasmic marker GFAP and (b) H2B-GFP and the astrocyte nuclear marker Pax2 (Figure 4, A and B). Quantification of the 2 nuclear markers, Pax2 and H2B-
GFP, shows greater than $99 \%$ correlation $\left(537 \mathrm{Pax}^{+} / \mathrm{H} 2 \mathrm{~B}-\mathrm{GFP}^{+}\right.$ cells and $5 \mathrm{Pax}^{+} / \mathrm{H} 2 \mathrm{~B}-\mathrm{GFP}^{-}$cells). A comparison of H2B-GFP accumulation in cells at the vitreal surface (color-coded green in Figure 4C) and cells in the neural retina (color-coded red in Figure 4C) shows that Cre-mediated recombination in astrocytes (large flat nuclei, color-coded green) is largely complete by P2 and that recombination in retinal cells (small compact nuclei, colorcoded red) increases between P2 and P4 but constitutes only a 
GSL DAPI

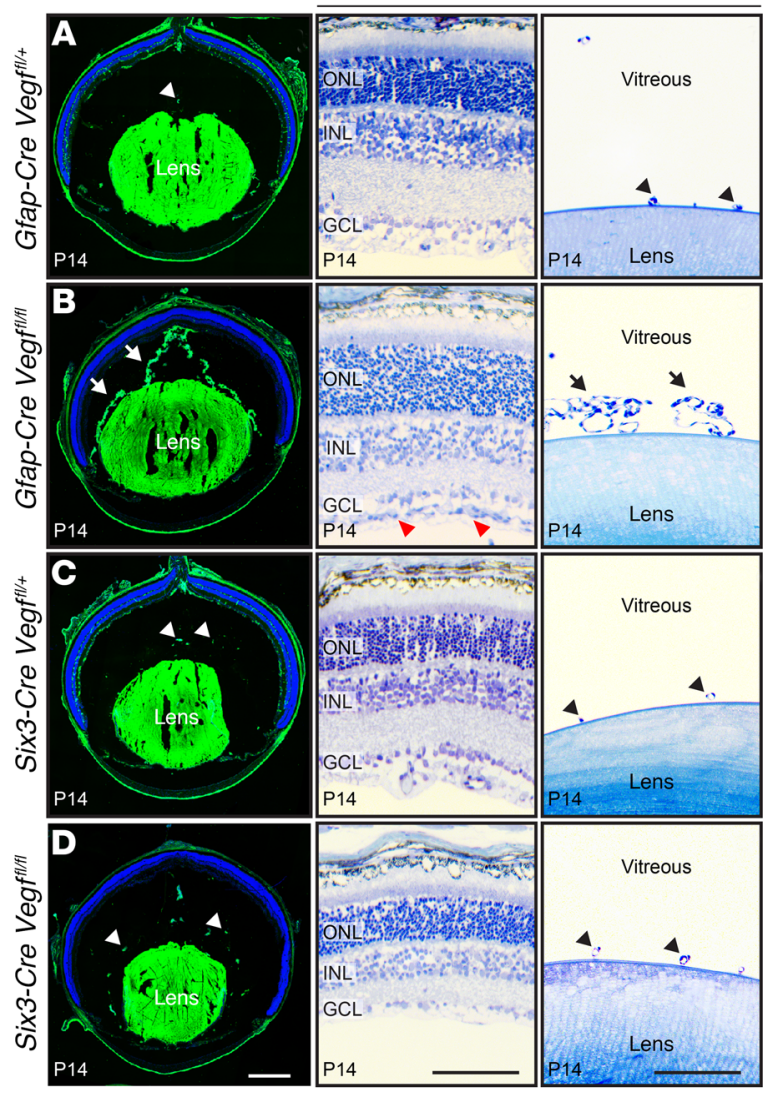

Figure 6. Persistent hyaloid vasculature in eyes without astrocytederived VEGF. (A-D) The left column shows frozen eye sections stained with GSL at P14. The hyaloid vasculature is retained in Gfap-Cre Vegfl/fl eyes (arrows in left panel of B) and regresses in Cfap-Cre Vegffl/+, Six3-Cre Vegff//+ and Six3-Cre Vegf $f^{f / f l}$ eyes (arrowheads in left panels of $\mathbf{A}, \mathbf{C}$, and $\mathbf{D}$ ). The right 2 columns show semithin plastic sections stained with toluidine blue (middle and far-right panels of A-D). In Cfap-Cre Vegff/ffl eyes, there is hyperproliferation of astrocytes on the vitreal face of the retina (red arrowheads in middle panel of $\mathbf{B}$ ) and retention of hyaloid vessels near the lens (retrolental vessels; arrows in far-right panel of $\mathbf{B}$ ). In the $\mathbf{3}$ other genotypes, the retrolental vasculature has almost completely regressed (arrowheads in far-right panels of A, C, and D). Representative frozen sections are shown from experiments with a total of 4 eyes per genotype, and representative plastic sections are shown from experiments with a total of 2 eyes per genotype. Scale bars: $500 \mu \mathrm{m}$ (left panels of $\mathbf{A}-\mathbf{D}$ ) and $100 \mu \mathrm{m}$ (middle and far-right panels of $\mathbf{A}-\mathbf{D}$ ).

small minority of retinal nuclei. In sum, these experiments indicate that the Messing Gfap-Cre line produces approximately $100 \%$ efficient Cre-mediated recombination in neonatal retinal astroctyes, as well as recombination in a minority of Müller glia and INL neurons starting several days later.

In contrast, using the same R26-LSL-mtdT-2A-H2B-GFP reporter to assess the Gutmann GFAP-Cre line, we observed only partial recombination in early postnatal (P1-P5) mouse retinas (Supplemental Figure 2). Thirty-six percent of astrocytes exhibited Cre-mediated recombination at P1 (429 $\mathrm{Pax}^{+} / \mathrm{H} 2 \mathrm{~B}-\mathrm{GFP}^{+}$ cells and $768 \mathrm{Pax}^{+} / \mathrm{H} 2 \mathrm{~B}-\mathrm{GFP}^{-}$cells). The fraction of recombined nuclei increased with age, but at P4 and P5, only $78 \%$ of astrocytes in the central vascularized retina were recombined (969 $\mathrm{Pax}^{+}$/

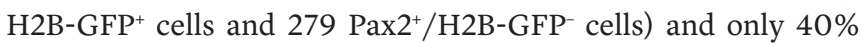

of astrocytes in the avascular peripheral retina were recombined (447 Pax2 $2^{+} / \mathrm{H} 2 \mathrm{~B}-\mathrm{GFP}^{+}$cells and $654 \mathrm{Pax}^{+} / \mathrm{H} 2 \mathrm{~B}-\mathrm{GFP}^{-}$cells). We think it likely that, in the context of the GFAP-Cre Vegfl/fl genotype, the relatively inefficient recombination of the Gutmann GFAP-Cre line leaves a sufficient number of astrocytes with at least 1 intact Vegf allele so that retinal vascular development is nearly normal. In the text and figures that follow, all Gfap-Cre experiments used the Messing line, and we will, therefore, simply refer to this transgene as Gfap-Cre.

We next asked whether retinas from Gfap-Cre Vegfl/fl mice exhibit defects in the development and/or migration of astrocytes or ECs. We also analyzed retinas from Six3-Cre Vegfl/fl mice in which loxP targets were recombined in all retinal neurons and astrocytes but not in ECs, beginning at approximately E9 (Supplemental Figure 3 and ref. 19). In Gfap-Cre Vegfl/fl retinas at P6, astrocyte proliferation and migration are indistinguishable from WT controls as determined by staining for Pax 2 and GFAP (astrocytes; Figure 5, A and B). By contrast, in Six3-Cre Vegfl/fl retinas at P6, astrocytes accumulate near the optic disc, indicating a defect in radial migration; it is not clear whether there is also a defect in astrocyte proliferation. In both Gfap-Cre Vegfl/fl and Six3-Cre $V e g f^{f / f l}$ retinas at P6, the retinal vasculature is dramatically attenuated, as determined by staining with GS-lectin, consistent with a severe defect in EC migration and/or proliferation (Figure 5, A and C). Contrary to the results of Scott et al. (12) and Weidemann et al. (13), this experiment shows that eliminating VEGF in developing astrocytes with a highly efficient Gfap-Cre driver inhibits radial migration of developing ECs despite the presence of a fully formed astrocyte network.

Figure 5D summarizes the astrocyte and EC phenotypes shown in Figure 3 and Figure 5A, together with inferences from these phenotypes regarding the role of VEGF signaling in early postnatal WT, $\alpha$-Cre Vegfl/fl, Six3-Cre Vegfl/fl, and Gfap-Cre Vegfl/fl retinas. As in Figure 2, in the schematics in Figure 5D (green nuclei) represent cells in which Cre-mediated recombination has occurred. In the developing WT retina, VEGF from astrocytes (blue arrows) is required to attract advancing ECs, and it may also act directly or indirectly to promote astrocyte migration (curved blue arrow with question mark). This inference follows from 3 observations: (a) eliminating VEGF in both retinal cells and astrocytes inhibits astrocyte migration (Six3-Cre Vegfl/fl diagram in Figure 5D), (b) eliminating VEGF from the neural retina but not from astrocytes permits astrocyte migration ( $\alpha$-Cre Vegflffl diagram in Figure 5D), and (c) eliminating VEGF from astrocytes, but not from the neural retina, also permits astrocyte migration, presumably because elevated levels of VEGF from the hypoxic retina compensate for the VEGF that would normally have been produced by astrocytes (Gfap-Cre Vegfl/fl diagram in Figure 5D). Importantly, in the presence of a fully formed astrocyte network, astrocyte-derived VEGF is both necessary and sufficient for radial migration of ECs, whereas retina-derived VEGF cannot drive radial migration of developing ECs (compare $\alpha$-Cre Vegfl/fl vs. Gfap-Cre Vegfl/fl diagrams in Figure 5D).

The action of astrocyte-derived VEGF on astrocyte proliferation and migration could be autocrine or paracrine (as shown in Figure 5D), but it could also include indirect actions on retinal cells, which then send secondary signals to astrocytes. As neural 
A

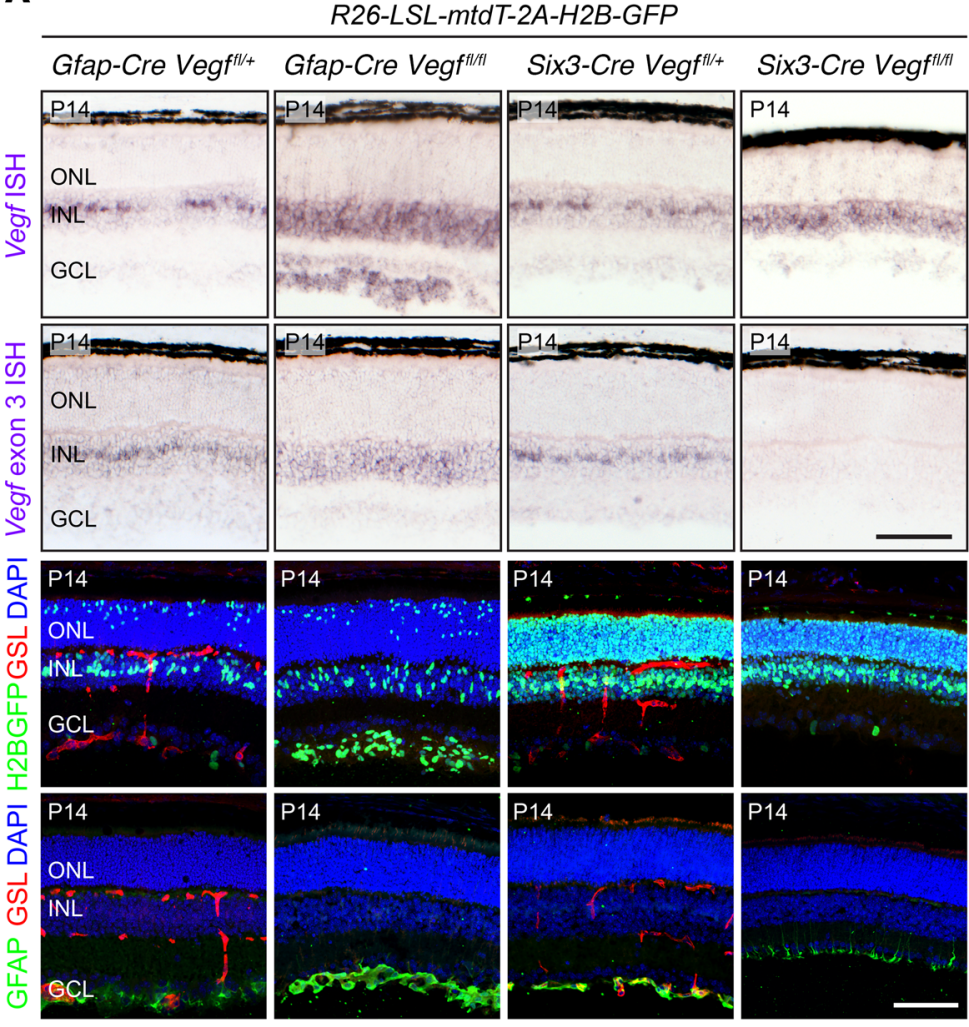

B

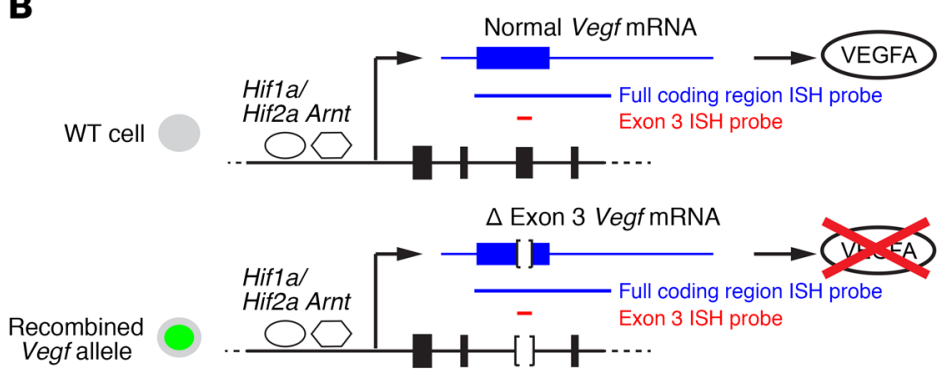

Figure 7. Contrasting responses of Vegf gene expression and astrocyte proliferation/migration with loss of Vegf in astrocytes versus all retinal cells. (A) Retina sections at P14 from mice carrying the R26-LSL-mtdT-2A-H2B-GFP reporter, Gfap-Cre or Six3-Cre drivers, and the indicated combinations of Vegf alleles. Top 2 rows show ISH for Vegf transcripts. First row, ISH with the full coding region probe detects transcripts from both the unrecombined and the recombined conditional allele. Second row, ISH with the exon 3-specific probe detects only unrecombined transcripts. Third row, localization of Cre activity visualized with the R26-LSL-mtdT-2A-H2B-GFP reporter, leading to production of H2B-GFP. Fourth row, astrocytes visualized with GFAP immunostaining. Retinal vasculature, visualized with GSL staining, is missing from Gfap-Cre Vegf fl/fl and Six3-Cre Vegf fl/fl retinas. Scale bars: $100 \mu \mathrm{m}$. (B) HIFstimulated transcription of the WT (top) and KO (i.e., exon 3 deleted; bottom) Vegf alleles in hypoxic cells and the structures of WT and exon 3-deleted Vegf transcripts. In the diagram of the Vegf gene, the first 4 exons are shown as black rectangles. The full coding region ISH probe is shown as a blue line and the exon 3-specific ISH probe is shown as a red line.

retina-derived PDGF-A provides a necessary signal for astrocyte migration by activating astrocyte-expressed PDGFR- $\alpha$ (7), we asked whether eliminating either astrocytic or retinal VEGF production affects astrocyte PDGFR- $\alpha$ expression. We observe that at P4, when astrocytes are proliferating and migrating across the retina, the levels of PDGFR- $\alpha$ in astrocytes were indistinguishable among WT, Six3-Cre Vegfl/ll, and Gfap-Cre Vegfl/ll mice (Supplemental Figure 4), suggesting that a decrease in PDGF signaling in astrocytes is an unlikely explanation for the astrocyte migration defect in the absence of VEGF.

Homeostatic responses of neuronal, glial, and vascular cells to retinal hypoxia. In response to retinal hypovascularization, regression of the hyaloid vasculature-a transient capillary network within the vitreous cavity-is delayed, presumably in response to an elevated level of retinaderived VEGF. (We use the phrase hyaloid vasculature to refer to all vessels within the vitreous body, including those adjacent to the lens, i.e., the retrolental vessels.) This phenomenon is seen in P14 Gfap-Cre Vegfl/fl eyes, which retain an extensive hyaloid vascular network; in contrast, phenotypically WT control Gfap-Cre Vegfl/t+ eyes exhibit normal hyaloid regression (arrows and arrowheads in Figure 6, A and B). At P14, both Six3-Cre Vegfl/fl and phenotypically WT control Six3-Cre Vegfl/t+ eyes show almost no remaining hyaloid vasculature (Figure 6, C and D). These different responses of the hyaloid vasculature in Gfap-Cre Vegfl/fl versus Six3-Cre Vegfl/fl eyes-both of which have avascular retinas-presumably reflect a high level VEGF production by hypoxic inner retinal neurons and Muller glia in Gfap-Cre Vegfl/fl retinas versus the absence of VEGF production by hypoxic inner retinal neurons in Six3-Cre Vegfl/fl retinas. In a control experiment, crossing Six3-Cre to the R26-LSL-mtdT-2A-H2BGFP reporter showed no Cre-mediated recombination in the lens, implying that the absence of hyaloid and retrolental vasculatures in Six3-Cre Vegfl/fl eyes reflects the loss of retina-derived Vegf.

To assess the retinal hypoxia response, we determined the location and abundance of Vegf transcripts by in situ hybridization in Gfap-Cre R26-LSL-mtdT-2A-H2BGFP Vegfl/fl and Six3-Cre R26-LSL-mtdT-2A-H2B-GFP $V e g f^{l / f l}$ retinas and compared the results with phenotypically WT control Gfap-Cre R26-LSL-mtdT-2A-H2B-GFP Vegfl/+ and Six3-Cre R26-LSL-mtdT-2A-H2B-GFP Vegfl/+ siblings at $\mathrm{P} 14$, when retinal vascular development is largely complete (Figure 7A). We also compared the pattern of Cre-mediated recombination and the growth and development of astrocytes and vasculature in retinas with the same 4 genotypes (Figure 7A). As shown in Figure 7B, Cre-mediated deletion of Vegf exon 3 from the Vegfl allele leads to the synthesis of an mRNA variant with an internal deletion, and this variant transcript is detected with the standard Vegf in situ hybridization probe, which encompasses the entire coding region and part of the $3^{\prime}$ untranslated region. In the phenotypically WT control retinas (Figure 7A, first and third columns), Vegf transcripts are concentrated in a subset of Müller glia cell bodies in the center of the INL and are also present at a lower level in many INL neurons. The variation in the spatial distribution of Vegftranscripts likely reflects anatomic heterogeneity in the INL and ONL capillary plexuses.

To assess the efficiency of recombination mediated by GfapCre and Six3-Cre, we used an in situ hybridization probe encom- 


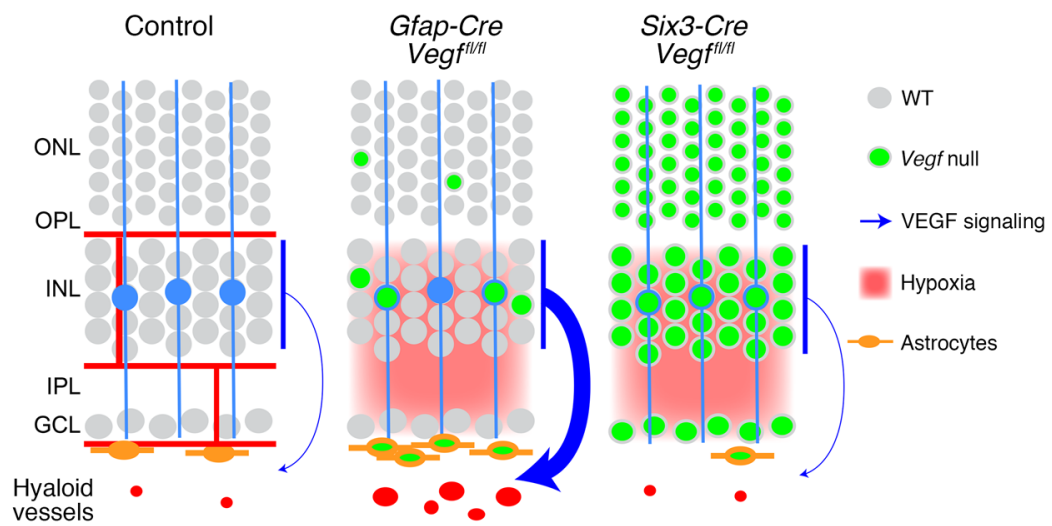

Figure 8. Retinal diagram as in Figure 2A showing the cellular sources, vascular targets, and relative strengths of VEGF signals inferred from the phenotypes of WT, Cfap-Cre Vegf $f^{f / f l}$, and Six3-Cre Vegfl/fl retinas. The persistent hyaloid vasculature in Gfap-Cre Vegfl/fl retinas is presumed to reflect high levels of retina-derived VEGF.

passing only Vegf exon 3, the exon in the Vegf conditional allele that is deleted by the Cre-mediated recombination (Figure 7B). In Gfap-Cre R26-LSL-mtdT-2A-H2B-GFP Vegfl/fl retinas, the exon 3 probe detected Vegf transcripts in the INL (where the vast majority of cells have not undergone Cre-mediated recombination) but not at the vitreal surface of the retina where astrocytes are located (Figure 7A). In Six3-Cre R26-LSL-mtdT-2A-H2B-GFP Vegfl/fl retinas, in situ hybridization signals with the Vegf exon 3 probe were below the limit of detection, consistent with Cre-mediated recombination in nearly all retinal cells.

In Six3-Cre R26-LSL-mtdT-2A-H2B-GFP Vegfl/fl retinas (Figure 7A, far-right column), Vegftranscripts (with exon 3 deleted) are only modestly elevated in the INL, a somewhat surprising result given the complete absence of hyaloid and retinal vasculatures as a source of oxygen. A likely explanation for this observation is suggested by the thinness of Six3-Cre Vegfl/fl retinas (compare middle panels in Figure 6, C and D; Figure 7A), which implies that severe inner retinal ischemia has impaired the growth, survival, and/or metabolism of retinal neurons. The Six3-Cre R26-LSL-mtdT-2A$H 2 B-G F P$ Vegfl/fl retinas also show a nearly complete loss of astrocytes (Figure 7A, lower right panel). The small amount of GFAP staining near the vitreal surface of these retinas represents Müller glial endfeet, which accumulate GFAP in response to retinal stress.

The most interesting observations relate to Gfap-Cre R26$L S L-m t d T-2 A-H 2 B-G F P$ Vegfl/fl retinas (Figure 7A, second column), which show a large increase in the number of astrocytes at the vitreal surface of the retina and an increase in Vegf transcripts throughout the inner retina, including in astrocytes (where both $V e g f^{l}$ alleles were recombined by Gfap-Cre) and in cells in the INL and ganglion cell layer (GCL) (where Gfap-Cre is expressed at a low level). Although Gfap-Cre R26-LSL-mtdT-2A-H2B-GFP Vegfl/fl retinas are completely avascular, they are of normal thickness and cellularity, most likely due to oxygenation by the persistent hyaloid vasculature (Figure 6B).

These data are summarized in Figure 8, which shows (a) the nearly complete elimination of VEGF production in Six3-Cre $V g^{f l / f l}$ retinas and the resulting loss of vitreal astrocytes and retinal and hyaloid vasculatures and (b) the expression of VEGF by
INL neurons in hypoxic Gfap-Cre $\mathrm{Veg} f^{f l / f l}$ retinas and the resulting hyperplasia of astrocytes and retention of hyaloid vasculature. The hyperproliferation of astrocytes observed on the surface of hypoxic Gfap-Cre R26-LSL-mtdT-2A-H2B-GFP $V e g f^{l / f l}$ retinas complements the observations of Zhang et al. (28) that astrocyte morphology is controlled by the oxygenation state of the retina. We note that tissue hypoxia can also affect the level and bioavailability of VEGF via posttranscriptional mechanisms that are independent of changes in Vegf transcript abundance (15).

Roles of VEGF and HIF in brain vascular development. Previous studies have described the severe prenatal neuronal and vascular defects resulting from early and widespread loss of Vegf or Hif-1a following inactivation of the corresponding conditional alleles with a Nestin-Cre driver (refs. 29-31, see also ref. 32 for analogous experiments in the avian embryo). We have extended these studies by analyzing the subtler phenotypes of Vegf and Hif- $\alpha$ KOs using Gfap-Cre, which recombines loxP targets in ventricular cells, radial glia, and glia associated with axon tracts in the fetal brain, as determined with the $R 26-L S L-m t d T-2 A-H 2 B-G F P$ reporter (Figure 9B).

Gfap-Cre Vegfl/fl mice are smaller than their phenotypically WT littermates; they die at approximately P20, and their brains show a dramatic reduction in the size and the thickness of the cerebral cortex, which appears semitransparent (Figure 9A). At E17 - an age when mutant and WT littermates are of equivalent size and therefore any differential in brain development is not secondary to a differential in overall health - the medial and dorsal cerebral cortex is thin and hypovascular (Figure 9B), but markers of cortical (Citp2 and Tbr1) and dentate gyrus (Prox1) neurons are unaffected (Supplemental Figure 5). These data are consistent with a model in which embryonic glia serve as a source of VEGF to promote cerebral angiogenesis in a manner analogous to the role played by retinal astrocytes.

Gfap-Cre Hif-1 $\alpha^{f l-}$ Hif-2 $\alpha^{f l-}$ mice have a milder and more variable phenotype than Gfap-Cre Vegfl/fl mice, generally surviving to adulthood and showing a smaller degree of cortical hypoplasia (Supplemental Figure 6A). In coronal sections of Gfap-Cre Hif-1 $\alpha^{f /-}$ Hif- $2 \alpha^{f l-}$ brains, the phenotype ranges from no discernable effect to a modest reduction in thickness in the medial and dorsal cortex. In Gfap-Cre Hif-1 $\alpha^{f l-}$ Hif-2 $\alpha^{f l-}$ brains with reduced cortical thickness, the vascular density is reduced to approximately $75 \%$ of its WT value (Supplemental Figure 6B). After weaning, Gfap-Cre Hif-1 $\alpha^{f /-}$ Hif-2 $\alpha^{f l-}$ mice gain weight marginally more slowly than their phenotypically WT littermates, but the difference is not statistically significant (Supplemental Figure 6C).

The similarity in location and appearance of the Gfap-Cre Hif-1 $\alpha^{f /-}$ Hif-2 $\alpha^{f l-}$ and Gfap-Cre Vegfl/fl cortical phenotypes is consistent with a model in which HIF-based sensing of hypoxia in cortical astrocytes titrates VEGF production, which functions as a major proangiogenic signal during cortical growth. The mild and variable phenotypes seen in Gfap-Cre Hif-1 $\alpha^{f l-}$ Hif- $2 \alpha^{f l-}$ mice may represent incomplete Cre-mediated recombination of the Hif-1a ${ }^{f l}$ or Hif- $2 \alpha^{f l}$ alleles, consistent with the data in Figure 1 showing that 
A

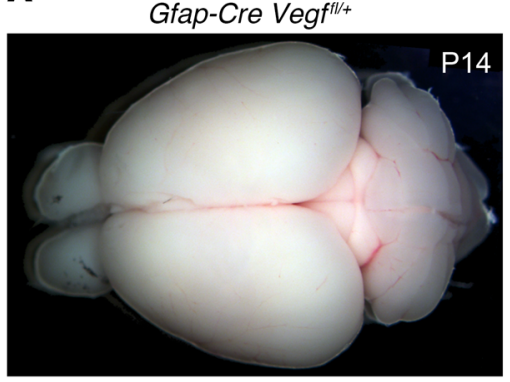

Gfap-Cre Vegf $f^{f / t I}$

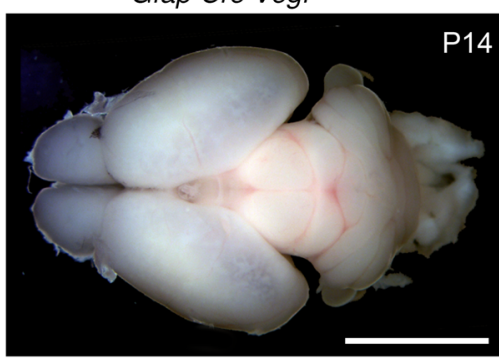

14
B

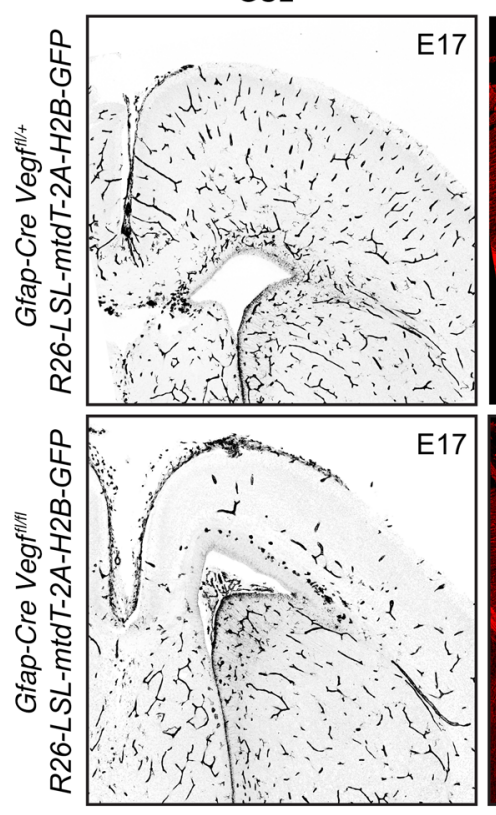

$\operatorname{mtd} T$
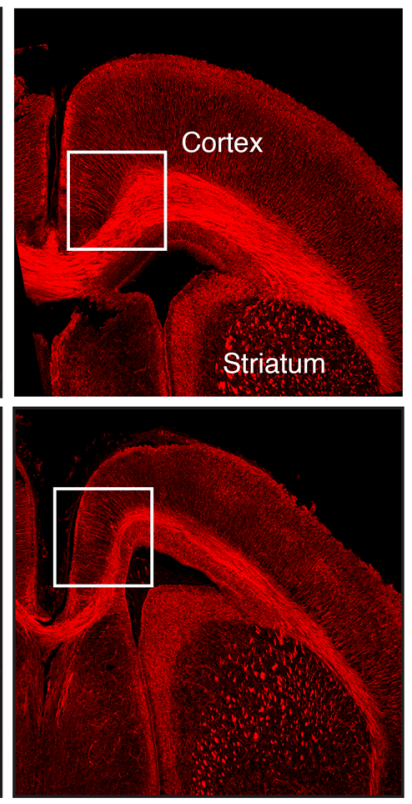
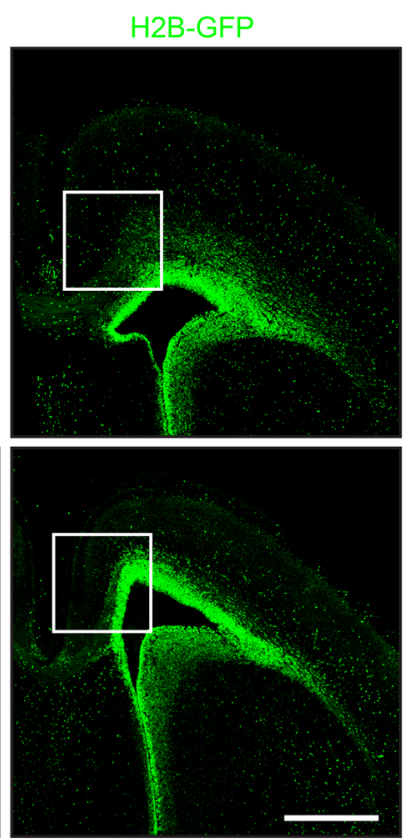
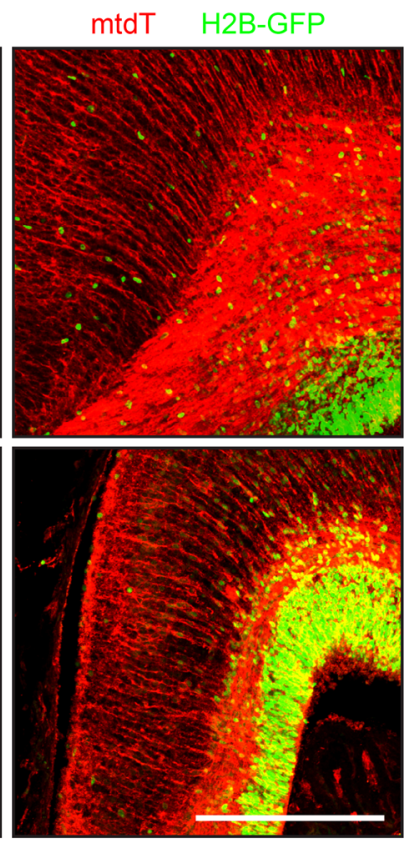

Figure 9. Loss of Vegf in radial glia and ventricular zone cells leads to hypovascularization and hypoplasia of the cerebral cortex. (A) Dorsal views of P14 brains. In the phenotypically WT Gfap-Cre Vegff/+ brain (left), the cerebral cortices cover most of the midbrain. In the Gfap-Cre Vegfl/fl brain (right), the cortex is hypoplastic and the midbrain is visible. Scale bar: $5 \mathrm{~mm}$. (B) Localization of vasculature (CSL staining; left panel) and Cre activity (right 3 panels) in coronal sections of E17 brains at the level of the anterior commissure. Cre activity, visualized with the R26-LSL-mtdT-2A-H2B-GFP reporter, shows mtdT and H2B-GFP in ventricular cells and radial glia. The Gfap-Cre Vegfl/fl dorsal cortex is thin and hypovascular. In the left 3 panels, the midline is near the left border of each image. Right panel shows enlargements of the regions delineated by the squares in the central 2 columns, with merged red and green channels. Scale bars: $500 \mu \mathrm{m}$ (left 3 panels) and $250 \mu \mathrm{m}$ (far-right panel).

recombination of all 4 alleles is required to generate a defect in retinal vascular development. Although the cortical thinning in Vegf- and Hif- $\alpha$-KO mice is most simply explained as a secondary effect of hypovascularization, a reduction in glia-derived VEGF could also have a direct effect on the development and survival of cortical neurons (33).

\section{Discussion}

In this study, we present a definitive genetic test of the role played by astrocyte-derived VEGF in the radial migration of ECs during retinal vascular development. The principal findings are as follows: (a) eliminating Vegf with a Gfap-Cre line that recombines in more than $99 \%$ of neonatal retinal astrocytes has no effect on astrocyte proliferation or radial migration, but it completely blocks radial migration of ECs; (b) eliminating Vegf with $\alpha$-Cre - which efficiently recombines in the progenitors of peripheral retinal neurons and Müller glia, but not in astrocytes-does not impair the proliferation or radial migration of astrocytes or ECs; (c) eliminating Vegf with Six3-Cre - which efficiently recombines in the progenitors of retinal neurons, Müller glia, and astrocytes-blocks the radial migration of both astrocytes and ECs. Taken together, these results lend strong support to the original model of Stone et al. (8), in which VEGF secretion by hypoxic astrocytes ahead of the growing vascular front drives radial migration of ECs. These results additionally point to essential and largely redundant actions of paracrine (retina-derived) and autocrine (astrocyte-derived) VEGF in the proliferation and migration of retinal astrocytes, as diagrammed in Figure 5D.

We also present a genetic analysis of the different roles of Hif-1 $\alpha$ and Hif-2 $\alpha$ in Müller glia versus neurons in the context of normal versus Norrin-deficient retinal vascular development. These results support and extend those of previous studies (16, 17, 22,24 ) and demonstrate that (a) the hypoxia response of retinal neurons is mediated by HIF-1 $\alpha$ and is required for development 
of the intermediate tier of capillaries, (b) the hypoxia response of Müller glia is mediated by HIF- $2 \alpha$ and is required for development of the outer tier of capillaries, and (c) the hyperproliferation of surface vasculature in $N d p^{K O}$ retinas requires an HIF-2 $\alpha-$ mediated hypoxia response (i.e., in Müller glia) and is not dependent on an HIF-1 $\alpha$-mediated hypoxia response (i.e., in neurons). These results highlight the distinctive roles of HIF- $1 \alpha$ and HIF- $2 \alpha$ in the context of normal and abnormal retinal vascular development, and they add to a growing body of data that points to the specialization of these 2 transcription factors in other biological contexts (34-36). The distinctive roles played by the hypoxia response - and, by inference, the production of VEGF-in different retinal cells could be relevant to understanding the response to intraocular administration of anti-VEGF biologics (37).

Finally, this study shows that cerebral cortical vascularization is diminished by Gfap-Cre-mediated deletion of Vegf in radial glia and ventricular cells, similar to the hypovascularization that results from Vegf deletion in retinal neurons and glia. Earlier work had demonstrated severe defects in vascularization and neural development in the early embryonic CNS resulting from widespread deletion of Vegf in CNS progenitors, neurons, and glia using a Nestin-Cre driver (29). The present results show that radial glia and/or ventricular cells serve as 1 essential source of VEGF.

Delineating the mechanisms of retinal vascular development is of special significance because this system has served as a paradigm for the analysis of molecular and cellular mechanisms in CNS vascular biology more generally (38). On both biological and technical grounds, the retina presents a uniquely favorable tissue in which to study CNS vascular development and disease, as enumerated here. First, the planar structure of the retina and the stereotyped anatomy of the retinal vasculature facilitate high-quality imaging and quantification of retinal arteries, veins, and capillaries. Second, the postnatal development of the mouse retinal vasculature and the nonlethal consequences of ocular defects make mouse retinal vascular development uniquely accessible to analysis and experimental manipulation. Third, mouse and human retinal vascular anatomy and vascular signaling pathways exhibit a close concordance, permitting the powerful tools of mouse genetics to be used for modeling human retinal vascular biology and disease. Fourth, due to the optical clarity of the eye, the retinal vasculature can be observed directly in living subjects, thereby permitting longitudinal noninvasive assessment of vascular microanatomy. With current technology, 3D images of the living human retina, including angiographic images, can be obtained at near-cellular resolution (39-41). Finally, the high degree of functional localization within the retina and the availability of quantitative psychophysical and electrophysiological tests of retinal function permit precise anatomic mapping and characterization of tissue dysfunction $(42,43)$. Together, these biological and technical attributes have paved the way for an increasingly sophisticated understanding of retinal vascular development, physiology, disease, and response to therapy.

\section{Methods}

Mice. The following mouse lines were used: $N d p^{K O}$ (ref. 44; JAX stock no. 012287); Pax6- $\alpha$-Cre ( $\alpha$-Cre; ref. 18); Six3-Cre (ref. 45; JAX stock no. 019755); Glast-CreER Tg (Slc1a3-cre/ERT, 1Nat/J; ref. 21; JAX stock no. 012586); Hif-1 $\alpha^{f l}$ (ref. 46; JAX stock no. 007561); Hif-2o $\alpha^{f l}$ (ref. 47; JAX stock no. 008407); $\mathrm{Tg}$ (Gfap-Cre) 25Mes/J (ref. 26; JAX stock no. 004600); Tg (GFAP-cre) 8Gtm (ref. 25; MGI ID: 2656248, provided by David Gutmann Washington University, St. Louis, Missouri, USA); $V_{\text {Vegfl }}$ (48); and R26-LSL-mtdT-2A-H2B-GFP (ref. 27; JAX stock no. 032577). All the mice used are of mixed genetic background except for the WT controls used in Supplemental Figure 1, which are C57Bl/6J. All of the other controls were siblings from the same crosses as were used to produce the experimental mice.

Genotyping primers. Genotyping primers are as follows: Vegfl $5^{\prime}$-loxP, AR1337 5'-CCTGGCCCTCAAGTACACCTTCCTGTCTC-3' and AR1338, 5'-GGAGCTGCCTCCGTACGACGCATTTCTAG-3'; Vegf $f^{l}$ 3'-loxP, AR1350, 5'-GATGGGTAGCTCTTAGGTAGCATTTAGG-3' and AR1350, 5'-GTGGAGATAGGAGGACCAGGGCTCTAAC-3'; Gfap-Cre, AR1382 (oIMR1900), 5'-ACTCCTTCATAAAGCCCT-3' and AR1383 (oIMR1901), 5'-ATCACTCGTTGCATCGACCG-3'; Pax6 $\alpha$-Cre, AR1348, 5'-AACCAATGAGGGCATTGCTGGCGTGGATAT-3' and JW412, 5'-ACCAGAGACGGAAATCCATCGCTCGACCAG-3'; GlastCreER, AR960, 5'-ACAATCTGGCCTGCTACCAAAGCTCTTCC-3' and AR961, 5'-CCAGTGAAACAGCATTGCTGTCACTTGG-3'; Hif-1 $\alpha^{-}$, AR1115, 5'-GCTACTGTAAATTTGGGGATGAAAACATCTGC-3' and AR1116, 5' 5 -GCAGTTAAGAGCACTAGTTGATCTTTCCGAGG-3'; Hif-1 $\alpha^{f l}$, AR1109, 5'-AAAAGTATTGTGTTGGGGCAGT-3' and AR1110, 5'-TGATGTGGGTGCTGGTGTCCAA-3'; Hif-2 $\alpha^{\prime}$, AR1101, 5'-CAGGCAGTATGCCTGGCTAATTCCAGTT-3' and AR1103, 5'-GCTAACACTGTACTGTCTGAAAGAGTAGC-3'; Hif-2 $\alpha^{\prime l}$, AR1104 (oIMR8468), 5'-GAGAGCAGCTTCTCCTGGAA-3' and AR1105 (oIMR8469), 5'-TGTAGGCAAGGAAACCAAGG-3'; Six3-Cre (generic Cre primers), JW411, 5'-TGCCACGACCAAGTGACAGCAATGCTGTTT-3' and JW412, 5'-ACCAGAGACGGAAATCCATCGCTCGACCAG-3'.

It is noteworthy that the first batch of $V e g f^{f / f l}$ mice that we acquired, referred to here as line 1 , showed no retinal vascular phenotype when crossed to $\alpha$-Cre mice. Careful genotyping revealed that these mice lack the 3' $\operatorname{loxP}$ site, downstream of Vegf exon 3, with the result that Cre-mediated deletion of Vegf exon 3 could not occur (Supplemental Figure 7). Vegfl/fl mice from a second source, referred to here as line 2, contain both loxP sites and these were used for the experiments described in this study. Using tail clips, genotyping of the unrecombined conditional allele and the recombined null allele was possible because Gfap-Cre is active in the skin of mice after approximately P7. If all cells in the body are heterozygous for the Vegfnull allele, the phenotype is embryonic lethality (48). To the best of our knowledge, there was only a single origin of the Vegfl allele, as described in Gerber et al. (48). We presume that the line 1 allele is derived from the original Veg $f^{n}$ allele and that it arose in a $V e g f^{1 /+}$ animal that had experienced a rare meiotic recombination event between the 2 loxP sites.

Antibodies and other reagents. Antibodies used in this study were as follows: rat anti-Ctip2 (ab18465 clone 25B6; Abcam), rabbit anti-Tbr1 (ab31949; Abcam), rabbit anti-Pax2 (71-6000; Thermo Fisher Scientific), goat anti-Prox1 (AF2727; R\&D Systems), rabbit anti-pimonidazole (PAb2627; NPI), rabbit anti-GFAP (Z0334, DAKO), and rat anti-CD140a (PDGFR- $\alpha, 558774$ clone APA5; BD Biosciences). Alexa Fluor-labeled secondary antibodies and GS-lectin (GSL; isolectin GS-IB4) were from Invitrogen. Hypoxyprobe (pimonidazole) was from NPI.

4-hydoxytamoxifen preparation and administration. Solid 4HT (Sigma-Aldrich H7904) was dissolved in an ethanol:sunflower seed oil (Sigma-Aldrich S5007) mixture (1:10 volume:volume) to a final 
concentration of $2 \mathrm{mg} / \mathrm{ml}$ and stored in aliquots at $-80^{\circ} \mathrm{C}$. For GLASTCreER experiments, 3 doses of $200-\mu \mathrm{g} 4 \mathrm{HT}$ were delivered by i.p. injection at $\mathrm{P} 6, \mathrm{P} 8$, and $\mathrm{P} 10$.

Tissue processing and immunohistochemistry. Tissue were prepared and processed for immunohistochemical analysis as described previously (49 and 50). Briefly, embryos and early postnatal brains were immersion fixed overnight at $4^{\circ} \mathrm{C}$ in $1 \%$ paraformaldehyde (PFA), followed by $100 \% \mathrm{MeOH}$ dehydration overnight at $4^{\circ} \mathrm{C}$. All tissues were rehydrated the following day in $1 \times \mathrm{PBS}$ at $4^{\circ} \mathrm{C}$ for at least 3 hours before embedding in 3\% agarose. For brains, 200-mm-thick tissue sections were cut using a vibratome (Leica). Fresh frozen eyes were sectioned at $14 \mu \mathrm{m}$, and the sections were fixed for 15 minutes at room temperature in 4\% PFA in PBS and then immunostained as described below. For retina flat mounts, eyes were fixed in 1\% PFA in PBS for 1 hour at room temperature, washed 3 times with PBS, and then the eye was opened and the retina gently dissected from the eyecup. After immunostaining, 4 or 5 radial incisions were made in the retinas so that it could be flattened on a microscope slide for imaging.

Tissue sections or intact retinas were incubated overnight with primary antibodies (1:200) PBSTC ( $1 \times$ PBS + 0.5\% Triton X-100 + $0.1-\mathrm{mM} \mathrm{CaCl}_{2}$ ) plus $10 \%$ normal goat serum. Tissues were washed 3 times with PBSTC over the course of 6 to 8 hours and subsequently incubated overnight with secondary antibodies (1:500) diluted in $1 \mathrm{x}$ PBSTC $+10 \%$ normal goat serum. Tissues were then washed at least 3 times with PBSTC over the course of 6 hours and flat mounted using Fluoromount G (EM Sciences 17984-25). Tissues were imaged using a Zeiss LSM700 confocal microscope using Zen Black 2012 software.

In situ hybridization to Vegf transcripts was performed as described previously (19). Vegf exon 3-specific sequence was amplified using primers $5^{\prime}$-TGATCAAGTTCATGGATGTC-3' and 5'-CTGCATGGTGATGTTGCTCT-3', and 3 copies were ligated in head-to- tail orientation into pBS-SK vector for riboprobe synthesis using T7 polymerase. Retinal sections from mice of different genotypes that were subjected to Vegf in situ hybridization were processed in parallel on a single slide and imaged with identical settings. Images were collected with a Zeiss Imager.Z1 microscope using AxioVision 4.9.1 software.

Processing tissue for plastic sections. Following cardiac perfusion with 2\% PFA and 2\% gluteraldehyde in PBS, eyes were immersion fixed in the same fixative overnight at $4^{\circ} \mathrm{C}$, treated for 90 minutes with $1 \%$ osmium tetroxide on ice, dehydrated in an ethanol series, embedded in Epon, sectioned at $0.5-\mu \mathrm{m}$ thickness, and stained with toluidine blue.

Image quantification. To quantify retinal vascular density, a set of $500-\mu \mathrm{m} \times 500-\mu \mathrm{m}$ square grid images were overlaid on a Z-stacked image of a GSL-stained flat-mounted retina or vibratome sections of brain as described previously $(19,51)$. Each grid consisted of 6 evenly spaced horizontal lines and 6 evenly spaced vertical lines $(6-\mathrm{mm}$ total line length per grid). The number of times that blood vessels and lines crossed was recorded for all grid lines and added up for each grid. A vessel with a trajectory coincident with a line for an extended distance was counted as crossing once for each coincident trajectory.
For the analysis of retinal vascular growth or morphology, 2-4 images were analyzed per retina, each in a separate quadrant, and these data are shown in the scatter plots and were used to obtain the $Q$ values shown in the figures. Supplemental Tables 1-9 also show the sample sizes and $Q$ values for comparisons in which (a) the data are averaged over the images of each retina and that average value is used as the input for the $Q$ value calculation, and (b) the data are averaged over the 2 retinas from each mouse and the average value for each mouse is used to input the data for the $Q$ value calculation. $Q$ values were calculated with the unpaired, unequal variance and 2-tailed Student's $t$ test, and adjusted for multiple comparisons using the method of Benjamini and Hochberg (52).

To quantify the density of the astrocyte process, images from anti-GFAP stained flat-mounted retina were scanned with identical exposure parameters. The fraction of GFAP-positive pixels in a series of 200- $\mu \mathrm{m} \times 200-\mu \mathrm{m}$ square regions was measured using the Fiji measure function in Image $(\mathrm{NIH})$.

Flat-mount retinas from P6 control (Six3-Cre Vegflt or Gfap-Cre Vegfl/+), Six3-Cre Vegfl/-, and Gfap-Cre Vegfl/- were stained with GSL and imaged with a Zeiss LSM700 confocal microscope at $\times 20$ magnification. The distance between the optic disc and the edge of the developing vasculature was measured using the measure function of Fiji in ImageJ (version 2.0.0). Two to 4 measurements in different radial directions were made on 5 to 8 retinas from 3 to 6 mice for each genotype, for a total of 14 measurements for each genotype.

Statistics. Statistical analysis was performed in MatLab (Mathworks), Microsoft Excel, and R (R Foundation for Statistical Computing, Vienna, Austria). Unpaired, unequal variance, 2-tailed Student's $t$ test was used to calculate $P$ values. $Q$ values were calculated using the Benjamini and Hochberg method (52).

Study approval. All mice were housed and handled, and experiments were performed with approval of the IACUC (protocol MO16M369) of the Johns Hopkins Medical Institutions.

\section{Author contributions}

AR and JN designed the study. AR conducted the experiments and collected the data. JW provided technical assistance. AR and JN analyzed the data. AR produced the figures. AR and JN wrote the manuscript.

\section{Acknowledgments}

The authors thank Martin Friedlander for the gift of Vegfl mice, David Gutmann and Shilpa Sanapala for the gift of Gfap-Cre mice, Yanshu Wang for helpful discussions, and Mark Sabbagh for helpful comments on the manuscript. This work was supported by the Howard Hughes Medical Institute, the National Eye Institute (R01 EY018637), and the Arnold and Mabel Beckman Foundation.

Address correspondence to: Jeremy Nathans, 805 PCTB, 725 North Wolfe Street, Johns Hopkins University School of Medicine, Baltimore, Maryland 21205. Phone: 410.955.4679; Email: jnathans@jhmi.edu.
1. Chung AS, Ferrara N. Developmental and pathological angiogenesis. Annu Rev Cell Dev Biol. 2011;27:563-584.

2. Semenza GL. Oxygen sensing, hypoxia-inducible factors, and disease pathophysiology. Annu Rev Pathol. 2014;9:47-71.

3. Selvam S, Kumar T, Fruttiger M. Retinal vasculature development in health and disease. Prog
Retina Eye Res. 2018;63:1-19.

4. Sun Y, Smith LEH. Retinal vasculature in development and diseases. Annu Rev Vis Sci. 2018;4:101-122. 
5. Fruttiger M. Development of the retinal vasculature. Angiogenesis. 2007;10(2):77-88.

6. Stahl A, et al. The mouse retina as an angiogenesis model. Invest Ophthalmol Vis Sci. 2010;51(6):2813-2826.

7. Tao C, Zhang X. Development of astrocytes in the vertebrate eye. Dev Dyn. 2014;243(12):1501-1510.

8. Stone J, et al. Development of retinal vasculature is mediated by hypoxia-induced vascular endothelial growth factor (VEGF) expression by neuroglia. J Neurosci. 1995;15(7 pt 1):4738-4747.

9. Gerhardt $\mathrm{H}$, et al. VEGF guides angiogenic sprouting utilizing endothelial tip cell filopodia. JCell Biol. 2003;161(6):1163-1177.

10. West H, Richardson WD, Fruttiger M. Stabilization of the retinal vascular network by reciprocal feedback between blood vessels and astrocytes. Development. 2005;132(8):1855-1862.

11. Usui Y, et al. Neurovascular crosstalk between interneurons capillaries is required for vision. JClin Invest. 2015;125(6):2335-2346.

12. Scott A, et al. Astrocyte-derived vascular endothelial growth factor stabilizes vessels in the developing retinal vasculature. PLoS One. 2010;5(7):e11863.

13. Weidemann A, et al. Astrocyte hypoxic response is essential for pathological but not developmental angiogenesis of the retina. Glia. 2010;58(10):1177-1185.

14. Duan LJ, Takeda K, Fong GH. Hypoxia inducible factor- $2 \alpha$ regulates the development of retinal astrocytic network by maintaining adequate supply of astrocyte progenitors. PLoS One. 2014;9(1):e84736.

15. Paredes I, Himmels P, Ruiz de Almodóvar C. Neurovascular communication during CNS development. Dev Cell. 2018;45(1):10-32.

16. Caprara C, Thiersch M, Lange C, Joly S, Samardzija M, Grimm C. HIF1A is essential for the development of the intermediate plexus of the retinal vasculature. Invest Ophthalmol Vis Sci. 2011;52(5):2109-2117.

17. Cristante E, et al. Late neuroprogenitors contribute to normal retinal vascular development in a Hif2a-dependent manner. Development. 2018;145(8):157511.

18. Marquardt T, Ashery-Padan R, Andrejewski N, Scardigli R, Guillemot F, Gruss P. Pax6 is required for the multipotent state of retinal progenitor cells. Cell. 2001;105(1):43-55.

19. Rattner A, Yu H, Williams J, Smallwood PM, Nathans J. Endothelin-2 signaling in the neural retina promotes the endothelial tip cell state inhibits angiogenesis. Proc Natl Acad Sci US A. 2013;110(40):E3830-E3839.

20. Raleigh JA, Miller GG, Franko AJ, Koch CJ, Fuciarelli AF, Kelly DA. Fluorescence immunohistochemical detection of hypoxic cells in spheroids and tumours. Br J Cancer. 1987;56(4):395-400.

21. de Melo J, et al. Injury-independent induction of reactive gliosis in retina by loss of function of the LIM homeodomain transcription factor Lhx2. Proc Natl Acad Sci U S A. 2012;109(12):4657-4662.

22. Rattner A, Wang Y, Zhou Y, Williams J, Nathans J. The role of the hypoxia response in shaping retinal vascular development in the absence of Norrin/ Frizzled 4 signaling. Invest Ophthalmol Vis Sci. 2014;55(12):8614-8625.

23. Mowat FM, et al. HIF- $1 \alpha$ and HIF- $2 \alpha$ are differentially activated in distinct cell populations in retinal ischaemia. PLoS One. 2010;5(6):e11103.

24. Ding K, Scortegagna M, Seaman R, Birch DG, Garcia JA. Retinal disease in mice lacking hypoxiainducible transcription factor-2 $\alpha$. Invest Ophthalmol Vis Sci. 2005;46(3):1010-1016.

25. Bajenaru ML, Zhu Y, Hedrick NM, Donahoe J, Parada LF, Gutmann DH. Astrocyte-specific inactivation of the neurofibromatosis 1 gene (NF1) is insufficient for astrocytoma formation. Mol Cell Biol. 2002;22(14):5100-113.

26. Zhuo L, Theis M, Alvarez-Maya I, Brenner M, Willecke K, Messing A. hGFAP-cre transgenic mice for manipulation of glial and neuronal function in vivo. Genesis. 2001;31(2):85-94

27. Wang Y, et al. Interplay of the Norrin and Wnt7a/ Wnt7b signaling systems in postnatal blood-brain barrier and blood-retina barrier development and maintenance. Proc Natl Acad Sci US A. 2018;115(50):E11827-E11836.

28. Zhang Y, Porat RM, Alon T, Keshet E, Stone J Tissue oxygen levels control astrocyte movement and differentiation in developing retina. Brain Res Dev Brain Res. 1999;118(1-2):135-145.

29. Haigh JJ, et al. Cortical and retinal defects caused by dosage-dependent reductions in VEGF-A paracrine signaling. Dev Biol. 2003;262(2):225-241.

30. Tomita S, et al. Defective brain development in mice lacking the Hif-1alpha gene in neural cells. Mol Cell Biol. 2013;23(19):6739-6749.

31. Raab S, et al. Impaired brain angiogenesis and neuronal apoptosis induced by conditional homozygous inactivation of vascular endothelial growth factor. Thromb Haemost. 2004;91(3):595-605.

32. James JM, Gewolb C, Bautch VL. Neurovascular development uses VEGF-A signaling to regulate blood vessel ingression into the neural tube. Development. 2009;136(5):833-841.

33. Mackenzie F, Ruhrberg C. Diverse roles for VEGF-A in the nervous system. Development. 2012;139(8):1371-1380.

34. Hu CJ, Wang LY, Chodosh LA, Keith B, Simon MC. Differential roles of hypoxia-inducible factor $1 \alpha(\mathrm{HIF}-1 \alpha)$ and HIF-2 $\alpha$ in hypoxic gene regulation. Mol Cell Biol. 2003;23(24):9361-9374.

35. Wang V, Davis DA, Haque M, Huang LE, Yarchoan R. Differential gene up-regulation by hypoxia-inducible factor-1 $\alpha$ and hypoxiainducible factor-2 $\alpha$ in HEK293T cells. Cancer Res. 2005;65(8):3299-3306.

36. Mole DR, et al. Genome-wide association of hypoxia-inducible factor (HIF)- $1 \alpha$ and HIF- $2 \alpha$ DNA binding with expression profiling of hypoxia-inducible transcripts. J Biol Chem. 2009;284(25):16767-16775.

37. Miller JW. Beyond VEGF-the Weisenfeld lecture. Invest Ophthalmol Vis Sci. 2016;57(15):6911-6918.

38. Usui Y, et al. Angiogenesis and eye disease. Annu Rev Vis Sci. 2015;1:155-184.

39. Drexler W, Fujimoto JG. State-of-the-art retinal optical coherence tomography. Prog Retin Eye Res. 2008;27(1):45-88.

40. Williams DR. Imaging single cells in the living retina. Vision Res. 2011;51(13):1379-1396.

41. Spaide RF, Fujimoto JG, Waheed NK, Sadda SR, Staurenghi G. Optical coherence tomography angiography. Prog Retin Eye Res. 2018;64:1-55.

42. Holder GE. Pattern electroretinography (PERG) and an integrated approach to visual pathway diagnosis. Prog Retin Eye Res. 2001;20(4):531-561.

43. Leinonen $H$, Tanila H. Vision in laboratory rodents - tools to measure it and implications for behavioral research. Behav Brain Res. 2018;352:172-182.

44. Ye X, et al. Norrin, Frizzled-4, and Lrp5 signaling in endothelial cells controls a genetic program for retinal vascularization. Cell. 2009;139(2):285-298.

45. Furuta Y, Lagutin O, Hogan BL, Oliver GC. Retina- and ventral forebrain-specific Cre recombinase activity in transgenic mice. Genesis. 2000;26(2):130-132

46. Ryan HE, et al. Hypoxia-inducible factor- $1 \alpha$ is a positive factor in solid tumor growth. Cancer Res. 2000;60(15):4010-4015.

47. Gruber M, Hu CJ, Johnson RS, Brown EJ, Keith B, Simon MC. Acute postnatal ablation of Hif-2 $\alpha$ results in anemia. Proc Natl Acad Sci U S A. 2007;104(7):2301-2306.

48. Gerber HP, et al. VEGF is required for growth and survival in neonatal mice. Development. 1999;126(6):1149-1159.

49. Wang Y, Rattner A, Zhou Y, Williams J, Smallwood PM, Nathans J. Norrin/Frizzled4 signaling in retinal vascular development and blood brain barrier plasticity. Cell. 2012;151(6):1332-1344.

50. Zhou Y, et al. Canonical WNT signaling components in vascular development and barrier formation. JClin Invest. 2014;124(9):3825-3846.

51. Rieder MJ, O'Drobinak DM, Greene AS. A computerized method for determination of microvascular density. Microvasc Res. 1995;49(2):180-189.

52. Benjamini Y, Hochberg Y. Controlling the false discovery rate: a practical and powerful approach to multiple testing. J R Stat Soc Series B Stat Methodol.1995;57(1):289-300. 